\title{
Assessing friction laws for simulating bowed-string motion
}

\author{
P. M. Galluzzo ${ }^{1}$, J. Woodhouse*1, and H. Mansour ${ }^{2}$ \\ ${ }^{1}$ Cambridge University Engineering Department, Trumpington Street, Cambridge CB2 \\ 1PZ, UK. \\ ${ }^{2}$ Computational Acoustic Modeling Laboratory, Schulich School of Music, McGill \\ University, 555 Sherbrooke Street West, Montréal, Québec H3A 1E3, Canada
}

August 4, 2017

\begin{abstract}
In order to carry out meaningful "virtual" experiments on the playability of bowed-string instruments, a simulation model is required that can reproduce all details relevant to a musician. Measured transient behaviour of machine-bowed strings is compared in detail with predictions from a range of previously-published computer simulation models. The general trends of waveforms and parameter dependence observed experimentally are successfully predicted, but some important details are not well captured by any of the models tested. The discrepancies, mainly associated with uncertainty about the correct model for the frictional interaction between bow and string, are examined systematically to reveal patterns of sensitivity to specific features of the models and to provide guidance on aspects of those models that may require enhancement to achieve a closer match to experiment. Of the models tested, the friction model based on contact temperature performed significantly better than more traditional ones based on instantaneous sliding speed.

PACS numbers:43.75.De
\end{abstract}

\section{Introduction}

There is a long history of theoretical modelling of the motion of a bowed string. In common with many other areas of science and engineering, such models were initially aimed at qualitative understanding of observed phenomena. More recently the focus has shifted to the use of computational models for "virtual testing", to supplement the slow process of making and testing prototypes to evaluate design changes. In the context of bowed instrument acoustics, a major aim of virtual testing is

*jw12@cam.ac.uk to learn something about "playability": those aspects of discrimination between instruments that can only be assessed by a player, not by a nonplaying listener.

There are several aspects to this question, which all depend upon the detailed response of a string to a given bowing gesture: the player's concern is "how easily can I make the string do what I want?", which could refer to achieving a particular regime of steady vibration or to details of transient response. The issue is often described with the phrase "ease of speaking". Perhaps computational models could help makers to produce instruments that are easier to play, by exploring design options with numerical experiments? Questions can be asked about the influence on the detailed string motion of various measurable features of the instrument body, strings, bow, rosin and player's gesture.

This idea goes back a long way, and preliminary efforts of this kind were made soon after the first computational models of bowed-string motion became available $[1,2,3]$. However, it is now known that those early models were insufficiently accurate for detailed studies, although they gave useful qualitative insights. Increasingly sophisticated theoretical/computational models of a bowed string have been developed since then, and these are now believed to capture many aspects of the underlying physics [4]. As will be seen in some detail later in this paper, the major remaining uncertainty concerns the physics of friction at the bow-string interface.

Two laboratory rigs have been built by different researchers with the specific intention of gathering data for testing and calibration of bowed-string models $[5,6]$. Many tests have been run on both, but only a few examples have been published. Although some years have passed since the measurements were made, these two rigs are still the best 
source of data for the purpose; but there has not yet been an attempt to survey all the available experimental data and compare it systematically with state-of-the-art simulations to assess the strengths and weaknesses of current models. That is the task of this paper.

A representative selection of data from the two test rigs will be compared with predictions of three specific models. These rigs and models will be described in the following sections. All simulations are based on a commonly-used computational strategy, and all aspects of these models and their numerical implementation are identical, apart from the treatment of friction. Questions of numerical implementation are not addressed here: the specific details have been previously published $[7,8]$. In summary, this paper makes no claims for novelty in the test rigs, development of theoretical models or numerical implementation. The novelty resides in the systematic comparison of model predictions with measured results, many of them previously unpublished. As will be seen throughout this paper, this comparison reveals that none of the models is fully satisfactory. It will provide some clues about directions for future development, and reveal tests that any improved model will need to pass.

\section{Background}

\subsection{Schelleng and Guettler diagrams}

There are several vibration regimes commonly encountered in bowed-string playing. The one a player is almost invariably aiming for was first described by Helmholtz [9], and is known as "Helmholtz motion". There is a single episode of sticking and of slipping between the bow and the string in every cycle of vibration, triggered by the passage past the bow of a fairly sharp "corner" (jump in slope) on the string. If the player does not press hard enough with the bow, more than one slip will occur in every period: "double-slipping" or more generally "multiple-slipping" motion. On the other hand, if the normal bow force is too high then some kind of non-periodic "raucous" motion is likely to occur.

Three less common regimes should also be mentioned. "Anomalous low frequencies" sometimes occur instead of raucous motion at high bow force; periodic motion with a much longer period than the string's natural period [10]. "S-motion" sometimes occurs when the bowing point on the string is near a position at a simple fraction of the string length (e.g. $1 / 3$ or $1 / 4$ ) [11]: it involves a single slip per cycle and is sometimes acceptable in mu- sical terms, but has a different frequency content from Helmholtz motion. Finally, "multiple-flyback motion" sometimes occurs when Helmholtz motion was intended: it involves two or more short slips in close proximity in every cycle, and produces a sound that is generally undesirable. Examples of waveforms associated with these regimes can be found in [3].

Which of these regimes occurs with a given bowing gesture, and in particular the combination of parameters needed to produce Helmholtz motion, is a question of great interest to players. Two famous diagrams have been used to convey partial answers. The first was suggested by Schelleng [12], who examined the limits of normal bow force $N$ within which it is possible to sustain steady Helmholtz motion. For a given value of the bow speed, both the minimum and maximum bow force limits depend on the position of the bow on the string, usually characterised via the parameter $\beta$ defined as the bow-bridge distance divided by the vibrating string length. Schelleng plotted the predictions of approximate formulae for the two force limits in the $N-\beta$ plane: on log-log scales the two lines define a wedgeshaped region within which Helmholtz motion may be sustained. An example is sketched in Fig. 1a. Since Schelleng's time, further developments of his analysis have been published [13, 1, 14, 15]: although some details of the theoretical bow force limits have been refined, the Helmholtz region qualitatively retains its wedge-like pattern.

However, the Schelleng diagram only addresses part of the problem. When an experienced player evaluates an unfamiliar instrument, they are primarily concerned with the transient response of the strings when various bowing gestures are performed. A natural candidate quantity to study, for possible correlation with a musician's evaluations of transient bowing, is the promptness of formation of the Helmholtz motion for a given bow gesture. Guettler [16] argued that for many simple bow gestures the force is kept almost constant while the bow accelerates roughly uniformly from rest. $\mathrm{He}$ thus suggested that an interesting parameter space in which to study transients would be the plane of bow force $N$ and bow acceleration $a$ : the "Guettler diagram".

Guettler analyzed the chain of events that occurs for the case of a constantly accelerating bow, to find out how a player might produce a "perfect transient" in which Helmholtz motion is achieved with no delay. His analysis, relying on a particular theoretical model of friction to be discussed in section 2.3 , led to the formulation of four conditions that must be satisfied by any perfect transient. Each of the four requires that the bowing 

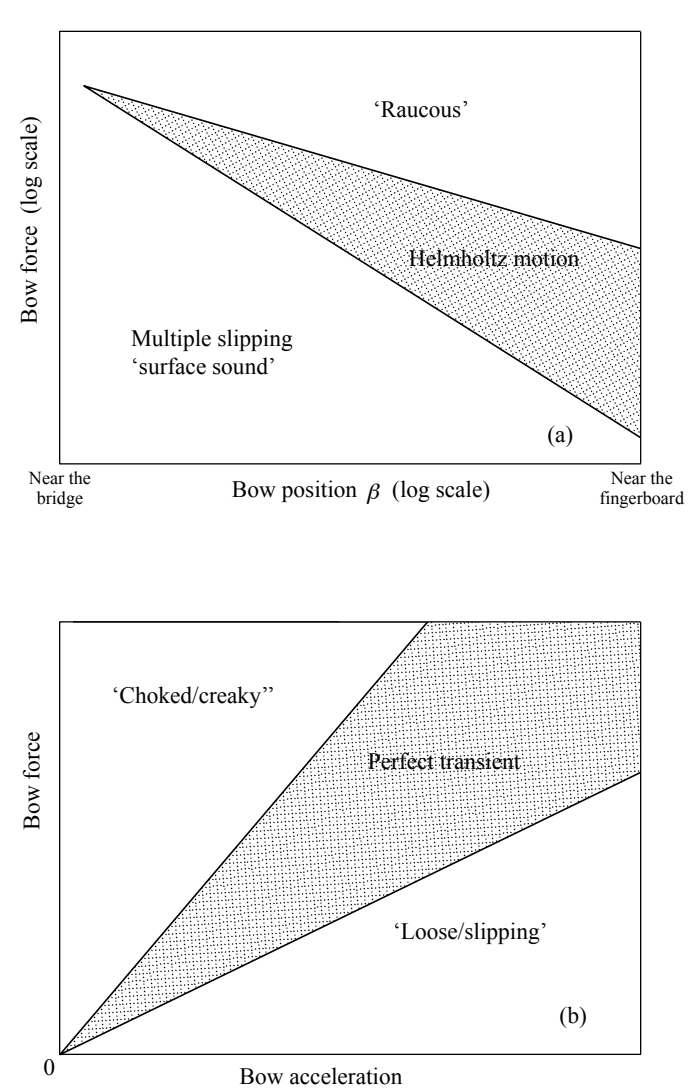

Figure 1: Sketches of the Schelleng (a) and Guettler (b) diagrams. Note that axis scales are logarithmic in (a), but linear in (b).

gesture lies to one side of a radial line from the origin in the $N-a$ plane, a different line for each condition. Some conditions require being above a line, others below one, so the region for which all four conditions are met occupies a wedge pointing toward the origin [16]. An example is sketched in Fig. 1b. If the bow force is too high or the acceleration is too low, the attack sounds "choked/creaky"; if the bow force is too low or the acceleration too high, the attack sounds "loose/slipping". As $\beta$ is decreased, i.e. as the bow is moved closer to the bridge, the wedge is predicted to become narrower and to rotate in a counter-clockwise direction in the plane [16].

\subsection{Test rigs}

Two sources of experimental data are used here: measurements by Galluzzo [6] on a cello string and measurements by Schumacher $[5,17]$ on a violin Estring mounted on a laboratory monochord rig.

Galluzzo's computer-controlled bowing machine, designed specifically to facilitate detailed compari- son between theory and experiment, has been described previously [6]. Through a combination of open-loop control, closed-loop feedback compensation and careful hardware design, the bowing machine can change bow acceleration with a response time of around $10 \mathrm{~ms}$ while maintaining constant bow force with an accuracy of $\pm 3 \%$. For the cases to be discussed here the machine was used to produce bowing gestures with constant acceleration, or tailored to elicit steady Helmholtz motion for exploring the Schelleng diagram.

The machine was used to bow an open D string mounted on a cello. A "Dominant" D-string was used throughout: physical properties of this particular string have been measured by previous investigators $[18,19]$. The string motion was monitored by measuring the transverse force exerted at the cello bridge with a piezo-electric sensor built into the bridge. Signal conditioning was provided by a charge amplifier with a low-frequency roll-off measured to begin at about $0.5 \mathrm{~Hz}$, which implies that the sensor system could accurately measure features with time-scales many times longer than the fundamental period of the string.

The second test rig, designed with a similar purpose, has been described by Schumacher et al. [5]. Control of the "bow" is less sophisticated than in the Galluzzo rig: it was carried on a belt-driven trolley, controlled using the manufacturer's software and hardware. A steel monofilament violin $\mathrm{E}$ string was bowed, the transverse forces exerted on the supports at both ends of the string were measured, using similar piezoelectric sensors to that used in the Galluzzo rig. An inverse calculation was then used to deduce the transient waveforms of string velocity and friction force at the bowing point [5]. This gives an advantage over the Galluzzo rig: the string motion and friction force at the bowed point relate very directly to the physics of friction, whereas the bridge force measured by Galluzzo is more indirect. Many of the parameter values for the properties of this string, needed for modelling purposes, were measured as part of the experimental procedure: the inverse calculation on which the measurement is based requires an accurate model.

All the experimental measurements to be presented here used rosin-coated rods for the "bow": the Galluzzo rig used an acrylic rod of diameter $13 \mathrm{~mm}$ while the Schumacher rig used a glass rod of diameter $6 \mathrm{~mm}$. The glass rod was dip-coated in rosin from solution, while the acrylic rod was coated by the usual violinist's method of rubbing the cake of rosin to transfer a film: the low thermal conductivity of acrylic allows this to work easily [20]. The use of a rod rather than a conventional 
violin or cello bow was deliberate: the theoretical models take their simplest form when the string is bowed at a single point, and this simplest case is a natural first target for validation studies.

The Galluzzo rig has been used to examine individual transients, and also to scan the Schelleng and Guettler diagrams. The Schumacher rig could only be used to produce individual transients, but it provided additional data: for example, runs have been done in which the ambient temperature was progressively raised to see the effect on the frictional behaviour [17], and it has also been possible to examine samples of the glass rods in the scanning electron microscope to give direct visual evidence about frictional processes [5].

\subsection{Bowed-string model}

Theoretical models of a bowed string share many features with models of other sustained musical instruments such as the clarinet, flute or trumpet $[21,22]$; and also with models for many engineering problems involving self-excited vibration, such as squeal of vehicle brakes [23, 24]. All these problems involve an approximately linear system with one or more resonances, often very complicated in its details, driven into vibration by interaction with a nonlinear element of some kind. Since any physical system must dissipate energy, some form of nonlinearity is necessary if sustained vibration is to occur. The nonlinear element can take many forms: for example, in a clarinet the flexibility of the reed results in a nonlinear relation between air-flow and pressure drop in the mouthpiece (see for example $[25,21])$. In a bowed string, the dominant nonlinearity is associated with the friction force at the bow-string contact.

For such problems, the linear part of the system is usually straightforward (if laborious) to model with sufficient accuracy, but the nonlinearity may be much harder to pin down. This is particularly true in the case of friction. There is a large body of literature relating to measurement and modelling of dynamic friction, in contexts as diverse as brake squeal and earthquake mechanics (see for example $[24,26])$. However, there is no existing model that can reliably capture frictional behaviour under all circumstances: this is still an area of active research [26].

The computational approach to be used in this study is based on a methodology developed some decades ago (see for example [21, 27, 28]). The linear system in this case consists of the string and instrument body, and a natural way to calibrate the linear model and test its accuracy is to study the plucked response of the string. A detailed descrip- tion of the model and its numerical implementation has been given elsewhere [7], including comparisons of simulations with plucked-string measurements. The version of the model used in the present study employs accurate implementations of the frequency-dependent damping, the wave dispersion and the torsional motion of the strings, and for the case of the Galluzzo rig it includes coupling to a realistic multi-resonance cello body. However, because the experimental results were all obtained with rods rather than normal bows, it was not necessary to include the coupling to bow-hair and bowstick dynamics. All parameter values used in the simulations to be reported here are listed in Table 1.

\subsection{Friction models}

Three specific models will be examined in this study, which differ only in the way that the friction force at the bowed point is calculated. All three friction models are based in one way or another on independent physical measurements using violin rosin, and all three have been described in earlier literature.

All early work on bowed-string dynamics made use of a very simple friction model. This model, often known as the "friction-curve model", relies on two assumptions. The first is the AmontonsCoulomb "law" that the friction force $F$ during sliding is proportional to the normal force, leading to the familiar notion of a coefficient of friction $\mu=F / N$. The second assumption is that this friction coefficient depends only on the instantaneous value of the relative sliding velocity between bow and string.

Both assumptions are open to question in the case of the bowed string. Amontons' law is generally understood to rely on the statistics of asperity contacts between rough surfaces (see for example Johnson [29]). This assumption may work reasonably well for a ribbon of bow-hair with multiple contacts [4], but with a rigid rod "bow" one might expect something more like the nonlinear Hertz contact law to apply [29]. Doubts over the velocitydependence assumption are deeper-rooted, as will be explored in some detail in the remainder of this paper.

Numerical values for the velocity-dependent friction coefficient of typical violin rosin were first measured by Lazarus [30], and similar results were later obtained by Smith and Woodhouse [20]. In both studies two rosin-coated surfaces were forced to slide with a range of constant relative speeds, and the friction force was measured. A good fit to the data of Smith and Woodhouse is given by the func- 
Table 1: String properties for the two tested strings, as used in all simulations. For definitions of the three loss coefficients, see [7]. The "finger-stopped" case of Fig. 12 was computed using the value of $\eta_{F}$ given in brackets.

\begin{tabular}{|c|c|c|c|c|}
\hline String & & & Galluzzo rig & Schumacher rig \\
\hline Frequency & & $\mathrm{Hz}$ & 146.8 & 693 \\
\hline Tension & & $\mathrm{N}$ & 111 & 72.5 \\
\hline Mass/unit length & & $\mathrm{g} / \mathrm{m}$ & 2.7 & 0.42 \\
\hline Bending stiffness & & $10^{-4} \mathrm{~N} / \mathrm{m}^{2}$ & 3.0 & 0.47 \\
\hline Characteristic impedance & & $\mathrm{kg} / \mathrm{s}$ & 0.55 & 0.175 \\
\hline Loss coefficients & $\eta_{F}$ & $10^{-5}$ & 23 (69 for stopped) & 5 \\
\hline & $\eta_{B}$ & $10^{-2}$ & 12.5 & 0.2 \\
\hline & $\eta_{A}$ & $1 / \mathrm{s}$ & 0.11 & 2 \\
\hline Torsional wave speed & & $\mathrm{m} / \mathrm{s}$ & 758 & 4620 \\
\hline Torsional Q factor & & & 45 & 30 \\
\hline Torsional impedance & & $\mathrm{kg} / \mathrm{s}$ & 1.8 & 0.61 \\
\hline
\end{tabular}

tion

$$
\mu=0.4 e^{\left(v-v_{b}\right) / 0.01}+0.45 e^{\left(v-v_{b}\right) / 0.1}+0.35
$$

where $v$ is the string velocity at the bowed point and $v_{b}$ is the bow speed, both expressed in $\mathrm{m} / \mathrm{s}$. Simulations based on this equation will be referred to as the "classical friction-curve model".

The prediction of Eq. 1 is plotted in Fig. 2. The vertical portion of each curve shows the range of possible forces during sticking. The curved portions show the variation of friction force during slipping: the sign is always opposite to that of the sliding speed because friction always opposes motion. For a bowed string the relative sliding speed is usually negative, but under some circumstances "forward slipping" can occur, and then the portion of the curve with positive values on the horizontal axis is relevant.

The two straight dashed lines in Fig. 2 illustrate an aspect of any friction-curve model that will be important for later discussions. For reasons explained in detail by McIntyre et al. [21], certain portions of a friction curve are inaccessible. At a given moment during the evolution of the string motion, the force and velocity are determined by the intersection of the friction curve with a straight line whose slope is inversely proportional to the normal force. When that slope is lower than the maximum slope of the friction curve, multiple intersections can occur: this is sometimes known as "Friedlander's ambiguity" [31]. The resolution is a hysteresis rule, illustrated by the dashed lines plotted in Fig. 2. At the end of a sticking episode, the force and velocity undergo large jumps; while at the end of a slipping episode the jumps are smaller.

Galluzzo suggested a significantly different form of the friction-velocity relation for violin rosin, from an alternative argument based on the jumps just discussed [32]. If a friction-curve model really is a correct description of the underlying frictional constitutive law, then it follows from the graphical construction sketched in Fig. 2 that at a slip event the jumps in velocity and friction force are directly related through the shape of the friction curve [33, 32]. Galluzzo measured the force drop at the bridge for the first slip, for a set of transients in a Guettler diagram, and then made use of a prior measurement of the limiting static coefficient of friction to deduce points on the friction curve. A fit to his results is the function

$$
\mu=0.4 e^{\left(v-v_{b}\right) / 0.7}+0.35
$$

This friction curve is also plotted in Fig. 2, and simulations based on this equation will be referred to as the "reconstructed friction-curve model". The shape of this friction curve is very different from that of Eq. (1), even though both are determined from experimental results obtained with the same rosin: note especially the difference of slope at low sliding speeds, and the different limiting coefficients of sticking friction. These differences give a strong indication that the friction curve model must have serious shortcomings. This is unfortunate, as most theoretical analyses of bowed-string behaviour, including Schelleng's and Guettler's bow force limits mentioned earlier, rely on this model [14]. 


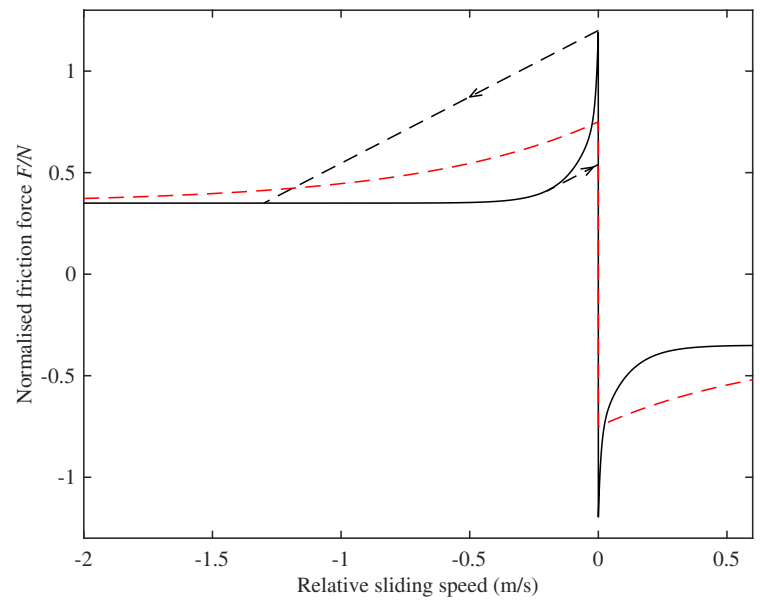

Figure 2: Friction curves used in this study. Solid black line: classical friction curve from Eq. 1; red dashed line: reconstructed friction curve from Eq. 2. Dashed straight lines illustrate the hysteresis rule which resolves the "Friedlander ambiguity" for the case of the classical friction curve [34] (see text). The loop is traversed in the direction indicated by the arrows.

Failure of models of the friction-curve type to slipping starts; rubbing of the two surfaces creates heat, softens the rosin and reduces the friction force; once the Helmholtz corner has moved away from the contact point, the disturbance force reduces and sticking recommences; heat loss through conduction results in reduction of contact temperature and the limiting friction coefficient increases again. The result is a kind of self-buffering behaviour, earlier studied for skis on ice [37]. Under conditions of steady sliding the contact temperature will increase with increasing sliding speed, and this would account for the type of variation captured in Eq. (1).

A preliminary attempt has been made to formulate a thermal model of friction that could be used in a bowed-string simulation. Two main simplifying assumptions were made. First, "contact temperature" was introduced as a single state variable. This was envisaged as representing the average temperature of the rosin within the active contact region, ignoring any spatial variation of temperature within this zone. It could be tracked by running a transient heat-flow calculation on a control volume of rosin in the contact region, in parallel with the dynamic simulation of the bowed string. At each instant the heat generated through friction is counterbalanced by advection, absorption and conduction, and a simple model of those processes was formulated [20].

The second assumption of the preliminary thermal model concerns a constitutive model for friction force as a function of temperature. Two general types of behaviour were explored: "viscous", with a temperature-dependent viscosity, and "plastic" with a temperature-dependent yield strength. In both cases, the material properties were not obtained from direct measurements but inferred by requiring that the combined mechanical/thermal simulation model should reproduce the measured steady-sliding behaviour, approximated by Eq. (1). Smith and Woodhouse found that out of the two models, the plastic model gave the better qualitative match to the stick-slip vibrations seen in their experiments, which were based on a vibrating cantilever beam rather than a string.

Woodhouse applied this "plastic thermal" friction model to simulate the bowed string, and compared the results to those of the classical frictioncurve model [18]. The thermal friction model was found to be more "benign" in the sense that the desired Helmholtz motion was established faster and more reliably than with the classical friction-curve model, at least with the particular parameter values used in the study. The reason for this qualitative difference of behaviour can probably be traced back to the fact that the thermal model never gives 
sudden jumps in force or velocity, unlike the friction curve models (see the discussion around Fig. 2 and the hysteresis rule). Jumps naturally lead to "twitchiness" of behaviour, in a similar way to the effect of saddle points in dynamical systems theory (see for example Glendinning [38]).

The purpose of the present paper is to compare the predictions of the thermal model and of the two friction-curve models with measurements on bowed strings. It is important to note that the two varieties of friction-curve model involve no free parameters, so they require no additional measurements to calibrate them. The thermal model is different in this regard. The physical quantity entering the model is the shear yield stress as a function of temperature, and to turn this into a friction force requires knowledge of the area of contact. This area will be governed by the contact geometry, which is different in the three relevant measurements. The steady-sliding calibration data was obtained with geometry somewhat similar to the Galluzzo rig, but with a larger area of contact. The Schumacher rig, with its much thinner string, has geometry that is different again, with a smaller contact area than either of the other experiments. This introduces an extra variable, the value of which is not accurately known by independent measurements. The influence of this additional variable will be discussed in section 4.2. Note that the contact area does not appear only through the conversion of yield strength to friction force - if so, it would be a simple scale factor - but it also influences the transient heatflow calculation that determines the evolution of contact temperature, so the overall effect is nonlinear and hard to guess without detailed simulations. Parameter values used in simulations with the thermal model are detailed in Table 2.

\section{Schelleng and Guettler dia- gram comparisons}

\subsection{Comparisons with simulations: Schelleng diagrams}

The result for Schelleng's diagram measured by the Galluzzo rig is shown in Fig. 3a. The bow speed was set to $0.05 \mathrm{~m} / \mathrm{s}$, the bow force was varied in twenty logarithmically spaced steps between the limits $0.1-3 \mathrm{~N}$, and the relative bow position $\beta$ was varied in twenty logarithmically spaced steps between the limits $0.02-0.18$. For details of the measurement procedure and the algorithm used to identify the regimes of string oscillation, see Galluzzo and Woodhouse [6]. Lines have been added to this plot to give a rough indication of the minimum and
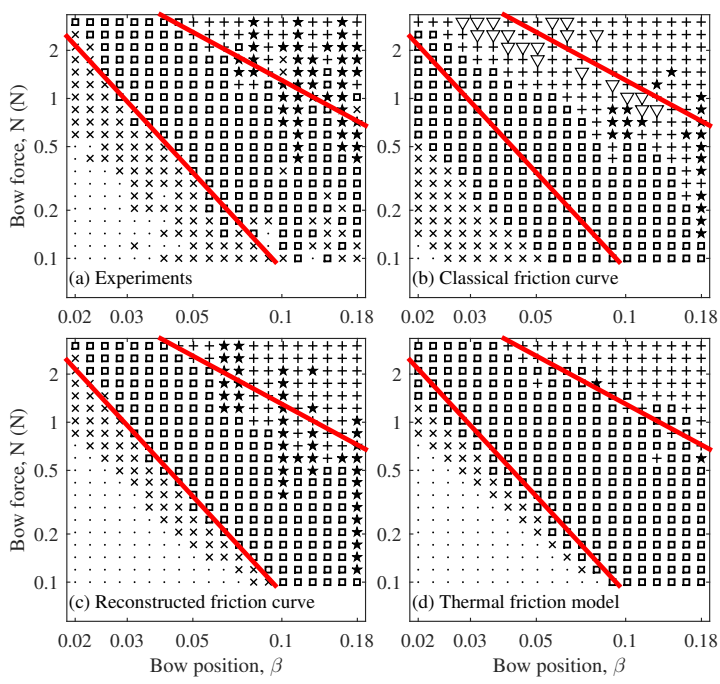

Figure 3: Schelleng's diagram: (a) as measured; (b) from simulation with the classical friction curve of Eq. (1); (c) from simulation with the reconstructed friction curve of Eq. (2); (d) from simulation using the plastic thermal model. Symbols denote the identified regime of oscillation - square: Helmholtz motion; $x$ : double or multiple slipping; dot: constant slipping; +: raucous motion; *: Smotion; triangle: anomalous low frequency (ALF). Straight lines indicate approximate upper and lower boundaries of Helmholtz motion in the measurements from (a), and are added to the other subplots as a guide to the eye when making comparisons.

maximum bow force limits in the measured data: these are simply intended as a guide to the eye. The same lines are reproduced in Figs. 3b-d, to help the reader make comparisons.

The Schelleng diagram shown in Fig. 3b was generated by simulation, using the classical frictioncurve model based on Eq. (1). The same values of bow position and force were used as in Fig. 3a. The regime identification was carried out using the same algorithm in all cases: see [18, 6] for details of the method. Figure 3c shows the corresponding Schelleng diagram generated with the reconstructed friction curve of Eq. (2). Finally, simulations were carried out using the thermal friction model. The resulting Schelleng diagram is shown in Fig. 3d, and is again directly comparable to the other plots.

Compared to the measurements, the Schelleng diagrams obtained from simulations with the friction-curve models, while different, exhibit recognisable similarities. Indeed, the case where the reconstructed friction curve was used matches some aspects of the measurements quite closely; particularly the position of the minimum bow force line. The Schelleng diagram obtained from sim- 
Table 2: Parameter values used in the thermal friction model. Thermal properties for the materials of rod and string were in all cases exactly as listed in Table 1 of [18]. Coulomb's law was assumed, so that the contact radius was proportional to the square root of the normal force, taking the listed values at the tabulated reference values of force.

\begin{tabular}{lcccc}
\hline & & Galluzzo rig & Schumacher rig & Steady sliding \\
Contact radius & $\mu \mathrm{m}$ & 250 & 200 & 500 \\
Reference force & $\mathrm{N}$ & 1 & 1 & 3 \\
Layer thickness & $\mu \mathrm{m}$ & 1 & 1 & 10 \\
\hline
\end{tabular}

ulations with the thermal model shows behaviour that echoes an earlier comment: it suggests more "benign" behaviour than the friction-curve models, possibly a little too benign. The Helmholtz region matches the red lines quite well, overflowing them a little at the edges, but the diagram lacks the conspicuous columns of S-motion seen in diagrams obtained from the measurements and also from simulations with the reconstructed friction curve.

To show what lies behind the symbols plotted in Fig. 3, Fig. 4 displays waveforms for column 10 of all four cases. The waveforms are separated vertically for clarity. Helmholtz motion is indicated by sawtooth waveforms. Towards the bottom of most columns instances of multiple slipping can be seen, and towards the top of all columns non-periodic "raucous" motion is seen. These waveforms give reassurance that the automated detection of vibration regimes that has been used to generate Fig. 3 has performed well: in all cases the plotted symbol corresponds to the judgement that would be made by eye. The plots suggest the same conclusion as the discussion above: the classical friction curve gives results considerably at variance with the measurements, while both the reconstructed friction curve and the thermal model give results that are recognisably similar to the measurements. Of the two, the thermal model looks slightly better, especially at higher normal forces.

\subsection{Comparisons with simulations: Guettler diagrams}

Examples of experimental Guettler diagram data for eight values of $\beta$, from [6], are shown in Fig. 5 . The values range from approximately $1 / 28$ (near the bridge) to approximately $1 / 6$ (near the fingerboard). The measurements for each case were made on a grid of $20 \times 20$ data points, linearly spaced in the $N-a$ plane. The chosen range of bow force was $0.4-3.2 \mathrm{~N}$, and the chosen range of bow acceleration was $0.08-3.2 \mathrm{~m} / \mathrm{s}^{2}$. In each plot, the time taken to achieve Helmholtz motion relative to the
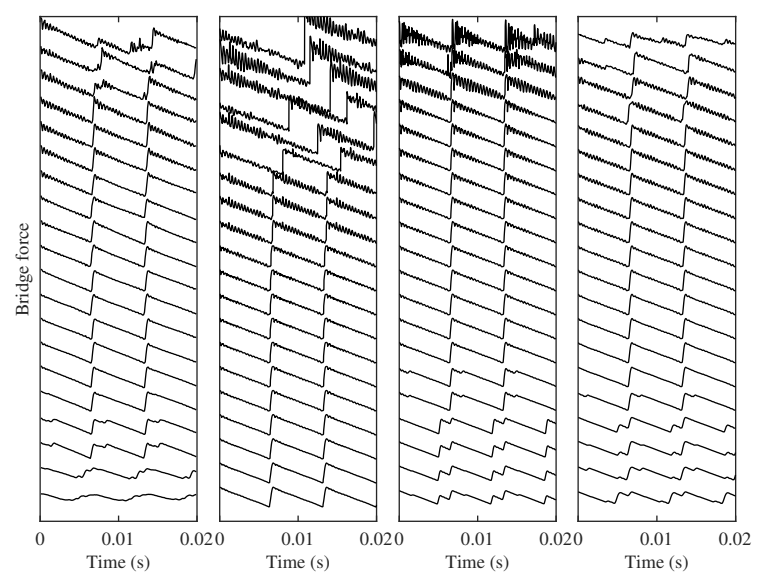

Figure 4: Bridge-force waveforms for the 10th column of Fig. $3(\beta=0.057)$. From left to right: measured, simulated with the classical friction curve, simulated with the reconstructed friction curve, and simulated with the thermal friction model. Curves are spread vertically for clarity, in the same pattern as in the Schelleng diagram.

time of the first slip at a given combination of bow force and acceleration is indicated by the shade of the pixel at the corresponding location in the $N$ $a$ plane. White pixels indicate perfect transients, black pixels indicate that it took twenty or more period lengths to achieve Helmholtz motion, and shaded grey pixels indicate intermediate cases. Pixels with crosses ("x") indicate unsuccessful measurements for which there were less than 20 string periods left in the recorded data after the first slip. The choice of 20 periods as the limit was simply to give a reasonable density of non-black pixels in the plots: it does not imply that a 20-period transient is necessarily short enough to be musically acceptable. Indeed, in the context of this particular note on the cello a 20-period transient would certainly be unacceptably long (see [39]). For an illustration of the algorithm used to determine transient length, see Fig. 9 of [6]; for more details of the implementation see [32]. 
The "speckly" texture of these plots may surprise a string player: a cellist experiences the string's response as fairly reliable and repeatable, whereas the intermingled dark and light pixels suggest that a small change in bowing gesture could have a big effect. It should be recalled that these results were generated with a carefully-controlled bowing machine and analysed with rigorous standards of what is acceptable as Helmholtz motion. It is not clear that all transients which "fail" by these tests are necessarily unacceptable in practice. Indeed, that kind of question goes to the heart of using such studies to assess "playability": there can be no doubt that further work will be needed to clarify the issue. As was reported in earlier work [6], when a given Guettler diagram scan was repeated under nominally identical conditions, the detailed light and dark pixels were not repeatable. However, the qualitative appearance of the diagram was repeatable: see especially Fig. 12 of that reference. That reference also showed that the speckly texture was not caused by the use of the perspex rod: a normal bow gave very similar results. When it comes to comparisons with simulated results, attention should be focussed on qualitative aspects: the position and shape of the general region within which non-black pixels occur, and the statistical nature of the "speckliness".

The Guettler diagrams shown in Fig. 6 were generated by simulation, using the classical frictioncurve model based on Eq. (1) just as was used for Fig. 3b. The results are directly comparable with the experimental results in Fig. 5: the same values of bow position, force and acceleration were used, and the length of pre-Helmholtz transient was determined using the same algorithm. Figure 7 shows corresponding Guettler diagrams generated with the reconstructed friction curve of Eq. (2), as was used for Fig. 3c. Finally, a corresponding set of Guettler diagrams simulated with the thermal friction model is shown in Fig. 8, as was used for Fig. 3d.

A comparison of Figs. 5-8 shows obvious differences. The reconstructed friction curve model performs significantly better than the classical friction curve model, as was previously observed with the Schelleng diagram, but the sparsity of nonblack pixels in both simulated cases would suggest a rather "unplayable" cello since relatively few combinations of bow force and acceleration can elicit Helmholtz motion in a reasonable time. The results for the thermal model show somewhat stronger similarities to the experimental measurements in terms of the overall positions and texture of the wedges of grey pixels in the Guettler diagram. As was seen with the Schelleng diagram, this model shows be-
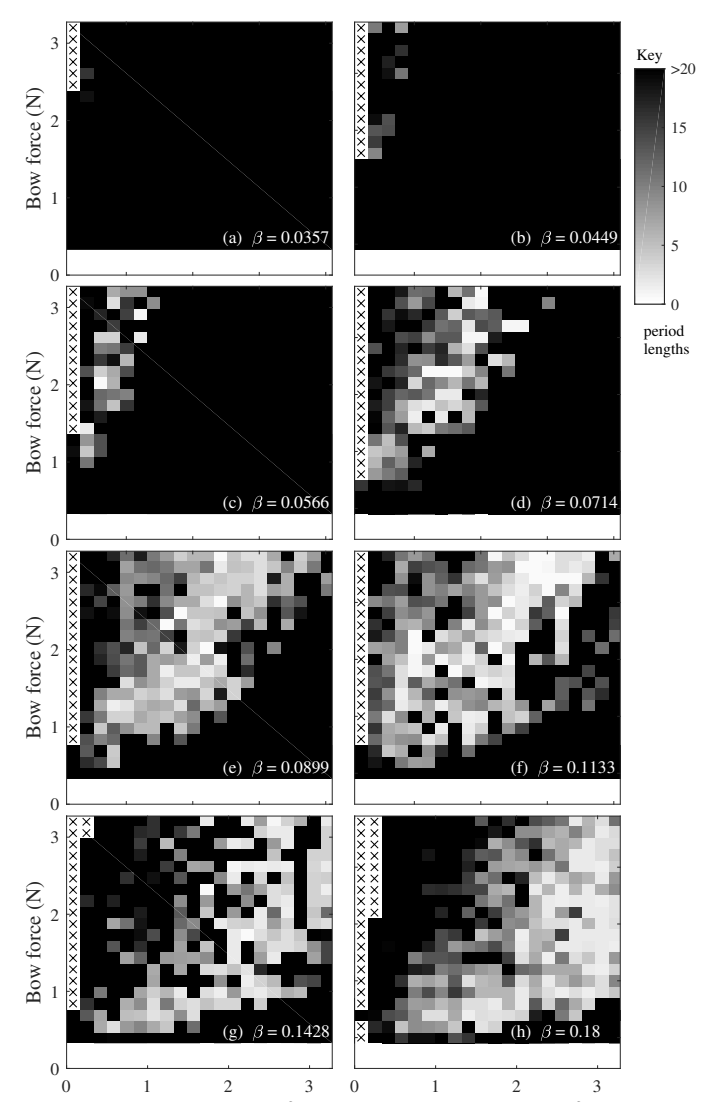

Bow acceleration $\left(\mathrm{m} / \mathrm{s}^{2}\right)$

Bow acceleration $\left(\mathrm{m} / \mathrm{s}^{2}\right)$

Figure 5: Experimentally measured Guettler diagrams, from [6], for eight different values of $\beta$ : from top left, $\beta=0.0357,0.0449,0.0566,0.0714,0.0899$, $0.01133,0.1428,0.18$. In each plot, the time taken to achieve Helmholtz motion relative to the time of the first slip at a given combination of bow force and acceleration is given by the shade of the pixel at the corresponding location in the $N$ vs. $a$ plane. White pixels with crosses (" $\times$ ") indicate unsuccessful measurements, as described in the text. The vertical and horizontal scales of each plot are the same. The top two plots are almost entirely black, because Helmholtz motion was rarely achieved in the allowed time with these values of $\beta$.

haviour that is rather more benign than the reconstructed friction curve model, and a great deal more so than the original friction curve model.

However, the thermal model certainly does not match experiments perfectly: for example, the upper and lower borders of the Helmholtz motion wedge appear to intersect the bow force axis at some distance above the origin, with the distance increasing as $\beta$ decreases. In the experiment, the intersection appears to be closer to the origin. The interpretation in terms of bowed-string behaviour is 


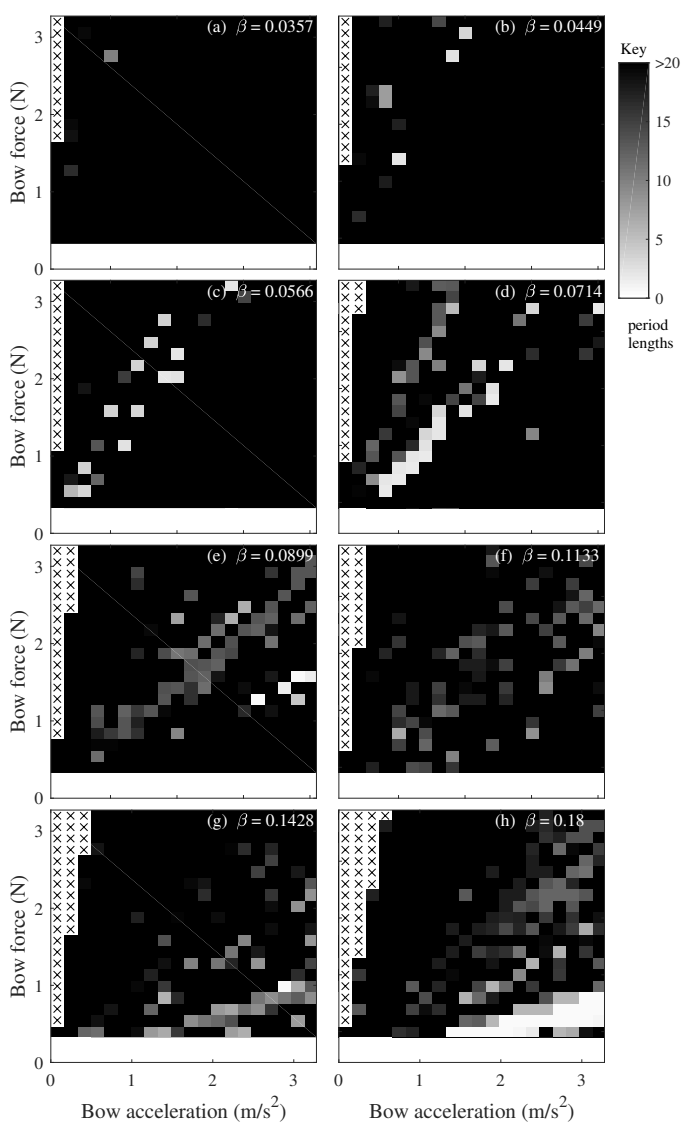

Figure 6: Simulated Guettler diagrams, for the same eight values of $\beta$ as in Fig. 5, with the classical friction curve model. In each plot, the time taken to achieve Helmholtz motion relative to the time of the first slip at a given combination of bow force and acceleration is given by the shade of the pixel at the corresponding location in the $N$ vs. $a$ plane, according to the colour guide on the right. This may be compared with the equivalent experimental measurements in Fig. 5, which were plotted using the same convention.

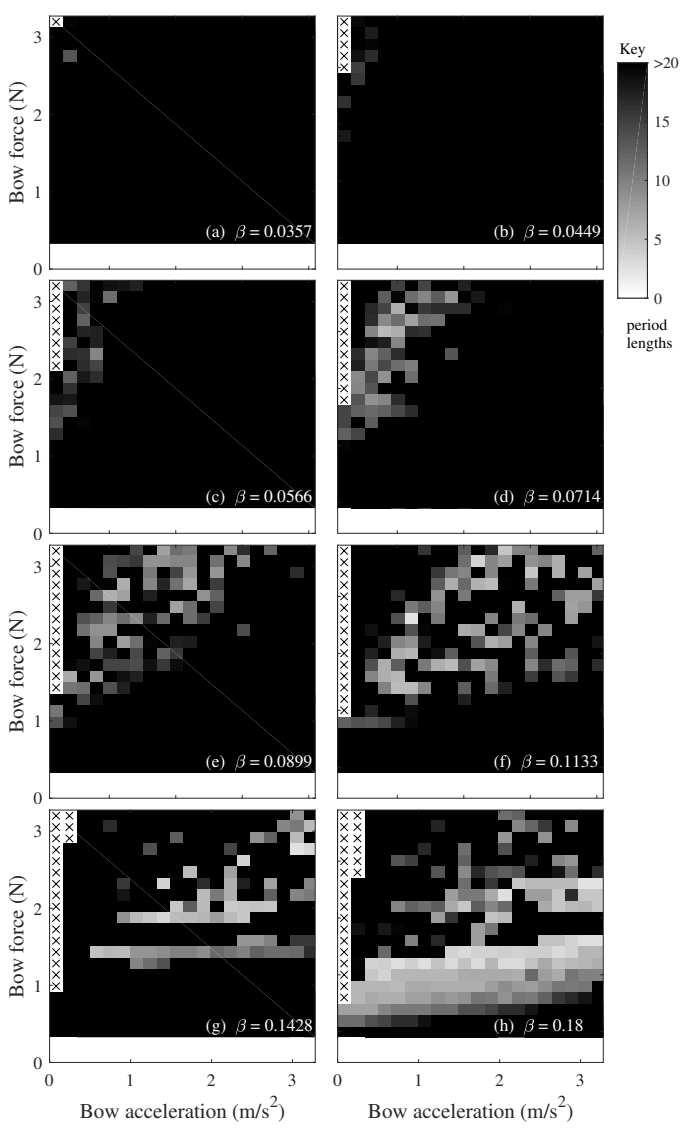

Figure 7: Same as Fig. 6, but the simulations are made using the reconstructed friction curve.

classical friction-curve model. The thermal model raises another set of questions, because it contains some parameter values that are not well determined by measurement. It is useful to know how sensitive the predictions are to these uncertain parameters. To get the clearest view of the answer to this question, it is examined in the context of steady motion and the Schelleng diagram without the added layers of complication associated with transients. Three parameters are explored in Figs. 9, 10 and 11. The first two relate to the assumed values of the rosin layer thickness and effective contact radius during the steady-sliding measurements on which the classical friction curve was based (values are given in Table 2; see [20, 18] for more details). The third is rather different: as mentioned earlier, it is far from clear that Coulomb's law would be expected to hold when a string is "bowed" by a rigid rod. The opposite limiting case would be represented by the Hertzian contact law (see for example Johnson [29]), in which the area of contact is proportional to $N^{2 / 3}$. The friction force would be expected to vary in a broadly similar way. 


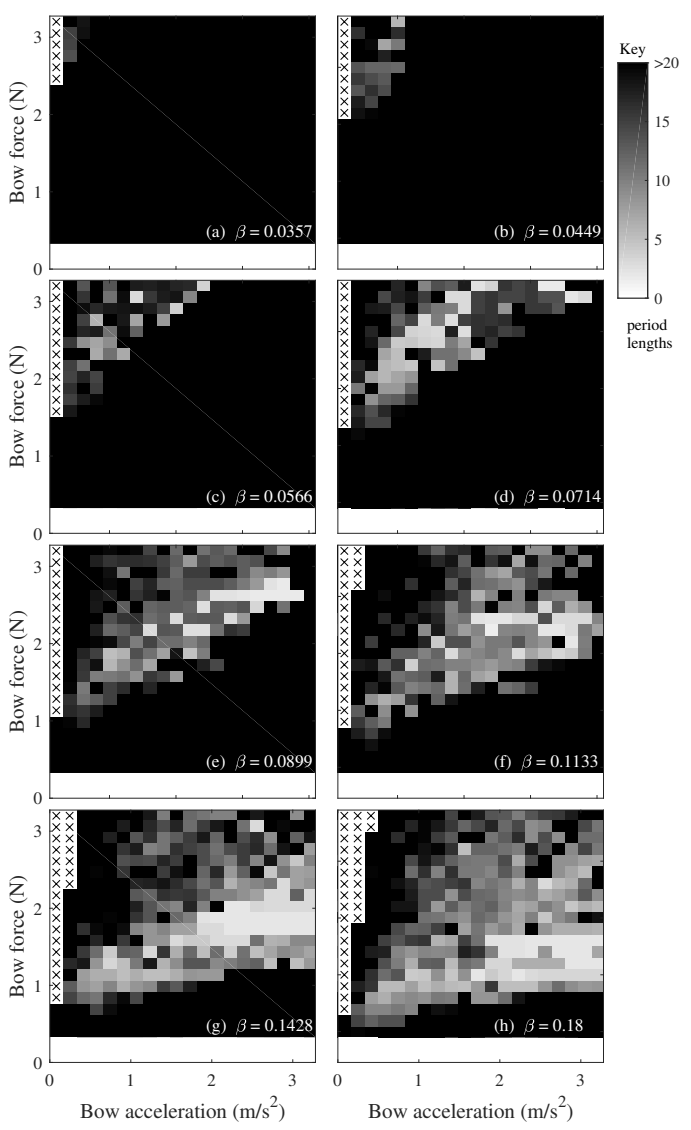

Figure 8: Same as Fig. 6, but the simulations are made using the thermal friction model.

The format of all three figures is similar: a modified case is compared to the assumed baseline case, and colours are used to highlight differences, particularly in the predicted extent of the Helmholtz motion range. Since the aim here is to show broad qualitative patterns, S-motion has been included with Helmholtz motion in these plots: the sporadic scatter of S-motion occurrences otherwise makes the plots less clear. Simulations have been carried out with finer resolution than in Fig. 3, because constraints associated with the resolution of the experimental tests are not relevant here. Figure 9 shows that the predictions are remarkably insensitive to the choice of assumed layer thickness. Figure 10 shows that a change in the reference contact radius has the effect of scaling the Helmholtz region up with relatively little change of shape or size: in this logarithmic plot the region is mainly shifted. This is not very surprising: one key use of the contact radius is to calculate the scale factor needed to convert the yield strength of the rosin into a friction force.

Figure 11 shows a more striking effect of the

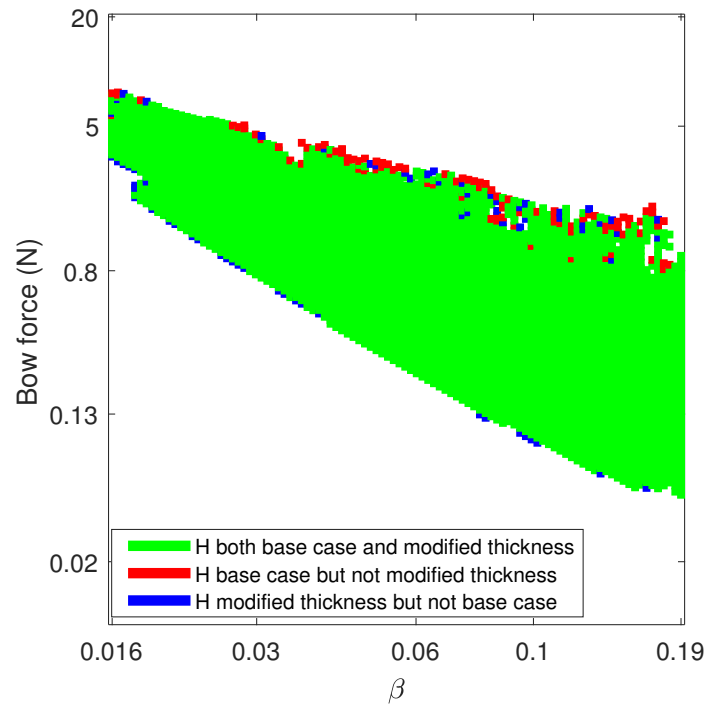

Figure 9: Modification to the Schelleng diagram, simulated with the thermal friction model, when the assumed reference layer thickness is changed from $10 \mu \mathrm{m}$ (base case) to $15 \mu \mathrm{m}$ (modified case). The colours indicate where Helmholtz motion or S motion, "H", was detected in either or both of the two cases: in this plot there is almost no difference between the two.

assumed contact law. With the Hertzian contact law, the Helmholtz region becomes bigger, and the slopes of the lines marking the minimum and maximum bow force change very significantly. The form of these bow force limits has been discussed quite extensively in earlier literature $[13,1,14,15]$, so it is perhaps a little surprising to find a new variable that has a very drastic effect on them. One can expect the real rod-string contact to show behaviour intermediate between Coulomb's and Hertz's laws. Hertz's law is based on an assumption of perfect smooth contact between the surfaces while Coulomb's law relies on a statistical population of asperities (see for example [29]). The actual contact conditions between the rod and string in the experiments are not known, but the contact footprint is very small to support a large number of asperities. However, it should be noted that Fig. 3 suggests rather good agreement between these particular experimental results and the thermal simulations based on Coulomb's law.

Some other model variations have previously been discussed in the context of steady motion and the Schelleng diagram [8], but it is now useful to examine the effect of these variations on transient motion via the Guettler diagram. Figure 12a shows a high-resolution $(300 \times 300)$ version of Fig. 6e, based on the classical friction curve model with 


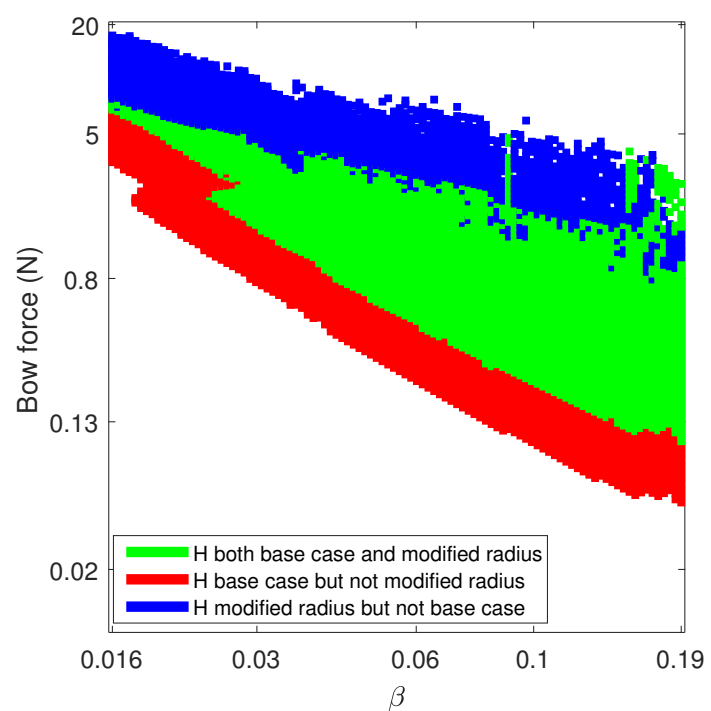

Figure 10: Modification to the Schelleng diagram, simulated with the thermal friction model, when the assumed reference contact radius is changed from $0.5 \mathrm{~mm}$ (base case) to $0.7 \mathrm{~mm}$ (modified case). The colours indicate where Helmholtz motion or S motion, "H", was detected in either or both of the two cases, using the same scheme as Fig. 9.

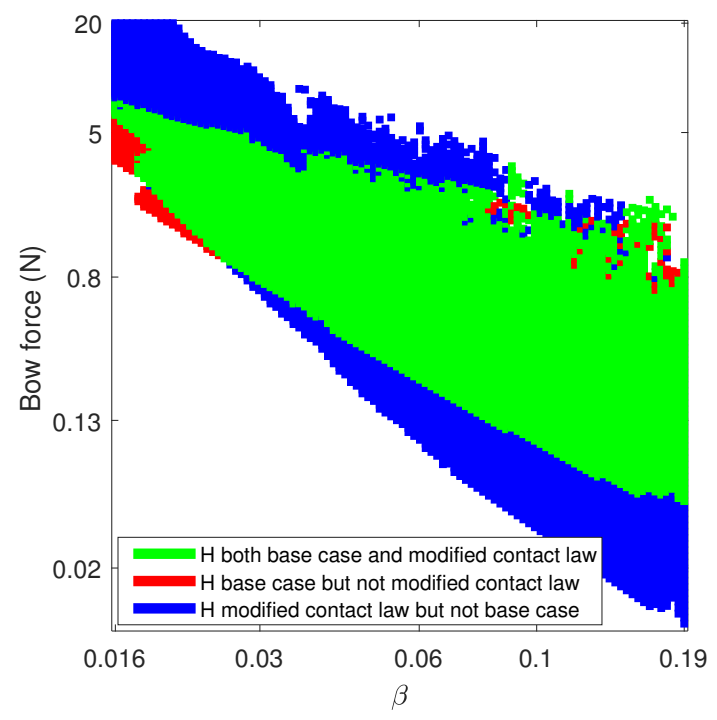

Figure 11: Modification to the Schelleng diagram, simulated with the thermal friction model, when the relation between normal force and friction force is changed from Coulomb's law (base case) to the Hertzian contact law (modified case). The colours indicate where Helmholtz motion or S motion, "H", was detected in either or both of the two cases, using the same scheme as Fig. 9. $\beta=0.0899$. Figure $12 \mathrm{~b}$ is the same as Fig. $12 \mathrm{a}$ except that the thermal friction model was used. Figures 12c-f show the effect of model variations within the classical friction-curve model on the appearance of the Guettler diagram. All the model parameters were kept the same as in Fig. 12a, except that in Fig. 12c the intrinsic damping of the string was increased to simulate a finger-stopped note (as opposed to an open string), in Fig. 12d the termination point of the string at the bridge was turned to a rigid boundary, in Fig. 12e the torsional motion of the string was excluded from the model, and in Fig. $12 \mathrm{f}$ the bending stiffness of the string was excluded from the model. The implementation of the "finger-stopped" case is based on empirical data [40]: the internal damping of the string was artificially boosted to match measured loss factors of a plucked string (see Table 1 for detailed values).

A striking feature of all cases except the thermal model in Fig. 12b, also apparent in Fig. 6e, is the presence of radial lines which contain apparently similar transients. Galluzzo has given an argument based on non-dimensional parameter groups, which predicts this radial structure [32]. However, as with Schelleng's and Guettler's calculations, the argument relies explicitly on the friction-curve model. The results here show that this restriction is important: the simulations using the thermal friction model do not show the radial structure, beyond a rather vague indication of the "Guettler wedge" containing all instances of Helmholtz motion. There is also no obvious sign of radial structure in the experimental data beyond the overall wedge shape of non-black pixels. This is another indication that friction-curve models of any kind give misleading predictions about transient response, an issue explored further in the next section.

Turning the bridge into a rigid termination reduces the number of Helmholtz samples and tends to make transients a little longer. By contrast, the "finger-stopped" case shows a larger number of Helmholtz occurrences and generally shorter transients. This observation is in accordance with the experience of the players, that a finger-stopped string is generally more playable than an open string [41]. Such a significant difference shows the importance of careful modelling of the string's damping. It may be an interesting topic for future work to explore whether the different sources of energy loss in a bowed-string system (intrinsic to the string or from the boundary conditions) have equivalent effects, or whether there are subtle differences.

Among the cases shown in Fig. 12 based on the classical friction-curve model, all but one show roughly a quarter of the samples classified as successful. The exception is the case with no tor- 


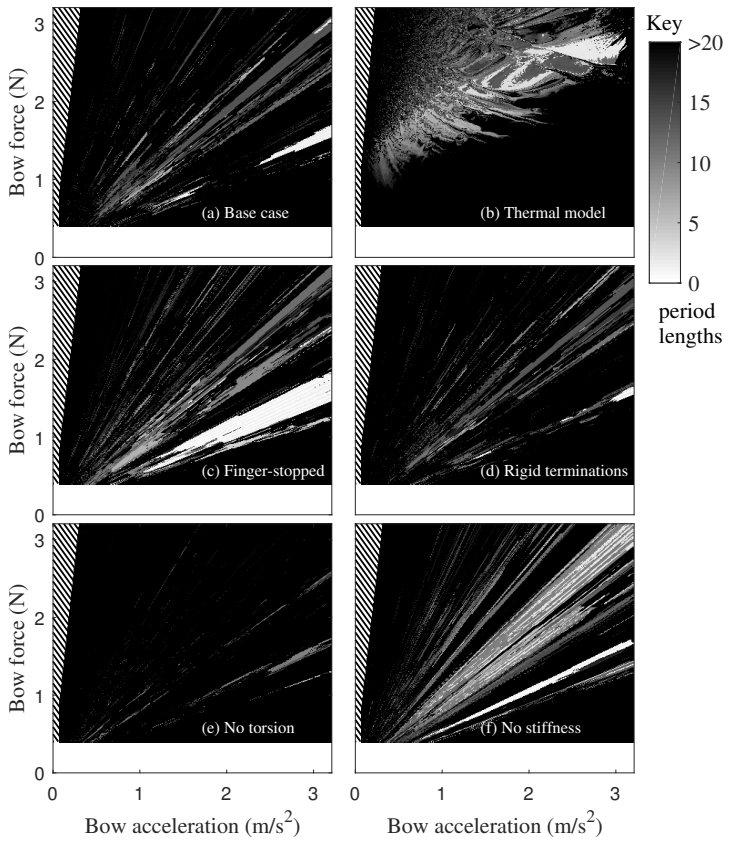

Figure 12: Simulated "Guettler diagrams" for an open $D_{3}$ cello string with different variations of the model. From top left: (a) the base case, using the classical friction curve model and $\beta=0.0899$; (b) as (a) but using the thermal model; (c) as (a) but for a finger-stopped string; (d) as (a) but assuming rigid terminations of the string; (e) as (a) but with torsional string motion excluded; (f) as (a) but for a string with zero bending stiffness. In each plot, the time taken to achieve Helmholtz motion relative to the time of the first slip at a given combination of bow force and acceleration is given by the shade of the pixel at the corresponding location in the $N$ vs. $a$ plane. Pixels covered by the hatched area indicate unsuccessful simulations, as described in the text. The vertical and horizontal scales of each plot are the same. sion, which shows a surprisingly small number of Helmholtz samples. The situation might have been eased to some extent if the less "twitchy" thermal friction model had been used. This conclusion contrasts with the findings of Serafin [42]: based on steady bowing results she reported remarkable insensitivity to the inclusion or exclusion of torsional motion. Note that Fig. 4 of [7] showed corresponding results for the Schelleng diagram using the present model implementation: the effect of excluding torsional motion was seen to be far from negligible, although less drastic than the influence on transients shown in Fig. 12e.

Finally, the case with no bending stiffness shows a larger number of successful samples, the average number of unsuccessful pre-Helmholtz periods is reduced, and the successful samples are more densely clustered around the center-line of the Guettler wedge. A denser clustering of successful transients is likely to correlate with an "easier to play" note from the player's point of view. However, in practice players have only limited control over the bending stiffness of their strings since they can only choose among the options offered by string manufacturers.

\section{Detailed comparison transient waveforms}

of

\subsection{Guettler transients}

The simulated Schelleng and Guettler diagrams, using all three tested models, have already shown clear deviations from the measured results. However, the high-level information obtainable from these plots does not give very clear indications of how friction models might need to be improved in order to obtain a better match. To get closer to the underlying physics requires a detailed examination of individual transient waveforms. A typical selection of measured and simulated bridge force waveforms from Guettler transients is shown in Fig. 13. The format is similar to Fig. 4: the measured results are on the left, and these are to be compared with the corresponding simulated results from the three models discussed earlier.

When the bow accelerates smoothly from rest, the string is effectively devoid of high frequency waves until the time of the first slip. In every case, measured and simulated, the force is observed to rise parabolically before the first slip, indicating that the string is displacing quasi-statically during that period. The constant acceleration a causes a string displacement at the bow $a t^{2} / 2$, and the lack of other waves on the string means that the bridge force, which is proportional to the slope of the displacement near the bridge, also increases in proportion to $a t^{2}$. (Strictly, the force at the bridge is also influenced by the string's bending stiffness, but normal musical strings are sufficiently flexible that this makes only a very small difference.)

For the measured results, if one assumes a maximum possible coefficient of friction $\mu_{s}$ associated with "limiting static friction", then a simple equilibrium force balance demonstrates that the bridge force just before the first slip must equal $(1-\beta) \mu_{s} N$. As noted earlier (see section 2.2), the sensor used to measure the bridge force had sufficiently good lowfrequency response that it could accurately measure features with time-scales as long as ten times the longest recorded pre-slip duration. It is safe to assume that bridge force measurements were not affected by electronic bandwidth limitations, and 888

889 
deduce the limiting coefficient of friction $\mu_{s}$ by dividing the measured bridge force just before the first slip by $(1-\beta) N$. Quantitative checks were made that the parabolic profile of measured bridge force did indeed match this prediction, with the known value of acceleration $a$.

One detail of Fig. 13 is worth commenting on immediately. The four cases show obviously different values of this "limiting sticking friction". The two friction-curve models simply follow the assumed coefficients of static friction, 1.2 and 0.75 for the two models (see Fig. 2). The thermal model was calibrated using the steady-sliding friction results, but it shows a lower value than the classical frictioncurve model for the maximum sticking friction because of the assumed value of the contact size (see Table 2). To illustrate the influence of this parameter within the thermal model, Fig. 14 shows alternative versions of the same set of simulated transients, with different assumed values for the reference contact radius. The induced changes are similar to those resulting from changing the normal force, but they are not exactly the same because the calculation of contact temperature also involves the contact radius. Notice from Fig. 13 that, with the radius chosen for the main simulations, the maximum friction force gives a reasonably good match to the experimental value: this was one consideration in choosing a suitable value for this radius.

Detailed waveforms vary within the Guettler plane, of course, and space does not allow the full set of results to be displayed. However, Fig. 13 correctly captures the main ingredients of the pattern. At the bottom of each stack of waveforms, with low normal force, string vibration grows only slowly. As force increases the response is faster, and for the highest forces some evidence can be seen of extended sticking during the early stages of a transient (most obviously in the simulations with the classical friction-curve model). These contrasting waveforms at high and low bow force correspond to extreme forms of Guettler's distinction between "loose/slipping" and "choked/creaky" responses to bowing.

The three models produce characteristically different patterns of response. The classical frictioncurve model tends to exhibit longer sticking than the others, both before the initial slip and later in the transients, and it shows bigger jumps in bridge force when slips occur. The reconstructed frictioncurve model tends to produce "fuzzy-looking" motion, involving a dense collection of smaller jumps in bridge force. This distinction is a natural consequence of the two shapes of friction curve: see the discussion of Fig. 2 and the hysteresis rule. For a given normal force, any friction-curve model

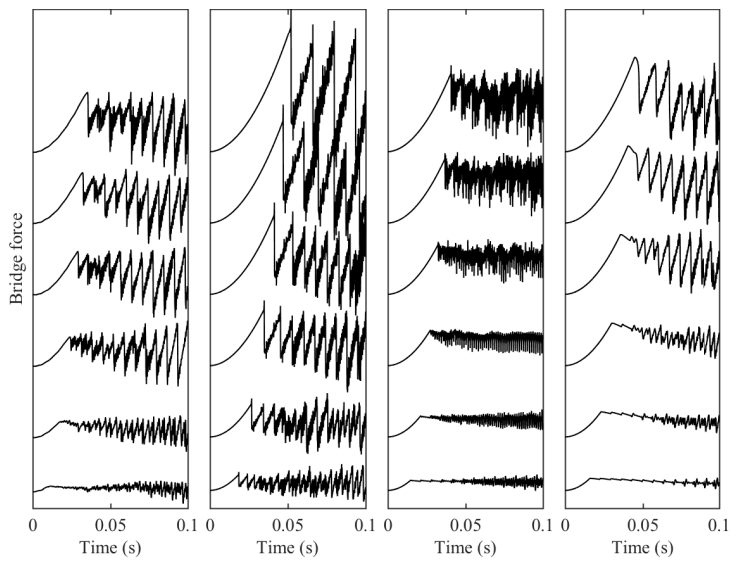

Figure 13: Selected bridge-force waveforms for the 10 th column of case (e) of the measured and simulated Guettler diagrams, Figs. 5-8 (acceleration $\left.a=1.56 \mathrm{~m} \mathrm{~s}^{-2}, \beta=0.0899\right)$. From left to right: measured, simulated with the classical friction curve, simulated with the reconstructed friction curve, and simulated with the thermal friction model. Curves correspond to rows 1, 4, 8, 12, 16 and 20 of the Guettler plots and are spread vertically for clarity.

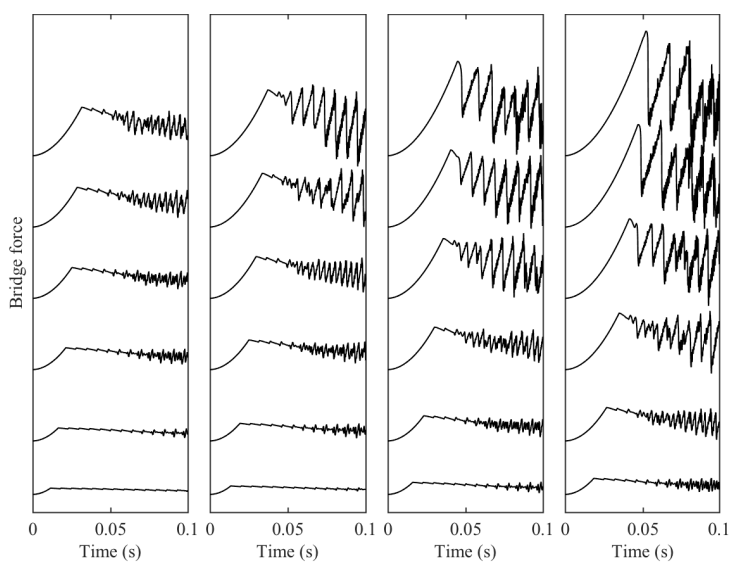

Figure 14: Alternative versions of thermal simulations matching Fig. 13, with different values of the assumed reference contact radius: from left to right, the values are $0.7,0.6,0.5$ and $0.433 \mathrm{~mm}$. The final value gives a "coefficient of sticking friction" of 1.2, matched to the classical friction-curve model. The value $0.5 \mathrm{~mm}$ was used in Fig. 13 and in all other simulations with this model. The values $0.5 \mathrm{~mm}$ and $0.7 \mathrm{~mm}$ correspond to the results for the Schelleng diagram in Fig. 10. 
may require a jump in friction force at the end of a sticking episode. The magnitude of these jumps is determined by the shape of the curve at low sliding speeds: the more dramatic shape of the classical curve leads to bigger jumps, the flatter form of the reconstructed curve gives smaller jumps.

The thermal model, by contrast, does not naturally produce jumps at all. Looking closely at the behaviour near the moment of first slip in each waveform of the right-hand set in Fig. 13, a rounded shape is always seen. The assumed model of plastic yielding at a stress dependent on temperature guarantees this. The yield stress, and hence the friction force, cannot begin to reduce until the temperature starts to rise, and temperature cannot rise until slipping starts and thus generates heat at the contact. The first slip is always a gradual process, leading to a rounded jump in friction force by a process of thermal runaway.

An overall comparison of the three simulations with the measurements, from Fig. 13, reinforces the earlier suggestion that none of the models faithfully reproduces all the details seen in the experimental data, but that the thermal model comes closest. However, from the specific point of view of the shape at the first slip this model behaves in a way that seems to be qualitatively wrong. The measured results show a definite jump at the first slip, at least for high values of normal force. At very low normal forces, jumps are hard to see: the bottom waveform in each group suggests that the reconstructed friction curve and the thermal model both mirror the measured behaviour fairly well, while the classical friction curve clearly does not.

The behaviour near the first slip seems to be giving valuable information about how a friction model needs to behave. The simple thermal model used here needs to be augmented in some way to allow for the possibility of a force jump while the rosin near the contact is still cold. Such a view is compatible with earlier discussion of scanning electron microscope images of the track left in a clean rosin surface by stick-slip events $[5,17]$. These tracks sometimes show direct evidence of heating and melting of the rosin, but in some cases they also showed evidence of brittle fracture. Fracture would naturally produce an abrupt jump in force. To guide future modelling effort, it is useful to extract more information about the first slip event from the measurements.

Figures 15 and 16 show results from an attempt to detect the first slip in each Guettler transient by an automated procedure, then record the magnitude of the maximum force before that slip, and the magnitude of the jump. The process of automated detection from noisy data is fallible: it relied on smoothing the data a little, differencing it, then testing for exceeding a hand-tuned threshold. Occasional rogue pixels in both plots show instances where this automated procedure failed, but many points were checked by hand and the reliability was verified to be generally good. Both plots reveal a very clear and systematic pattern of variation.
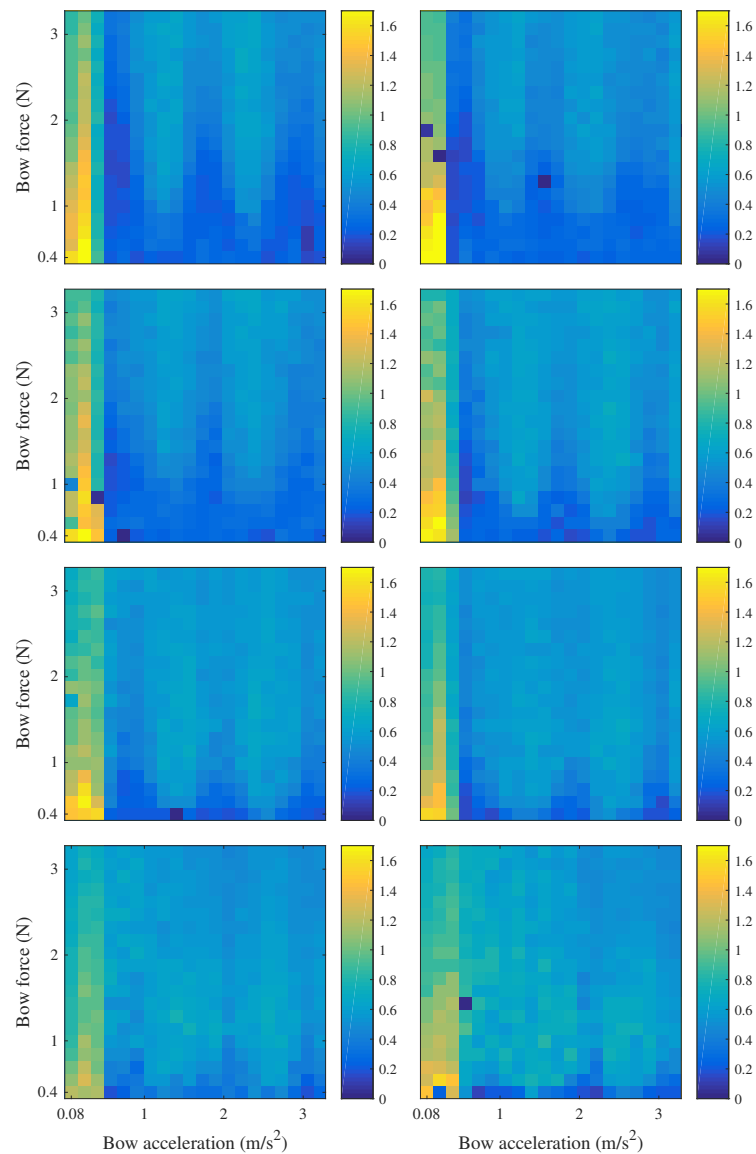

Figure 15: Maximum coefficient of friction before first slip (see text) based on the measurements shown in Fig. 5 and plotted in the Guettler plane for the same 8 cases as that figure. From top left, $\beta=0.0357,0.0449,0.0566,0.0714,0.0899,0.01133$, $0.1428,0.18$.

A preliminary simple observation about the results in Fig. 15 is that the average magnitude of the limiting static coefficient of friction is 0.66 across all the measurements (including others not shown here). With the classic friction curve model, and with the plastic thermal model at ambient temperature (at the start of a transient), the limiting static coefficient of friction is much bigger, at 1.2. This model value of 1.2 was derived from experimental measurements made under different conditions, and the difference with observations here gives a clear warning about over-generalising interpretations of 

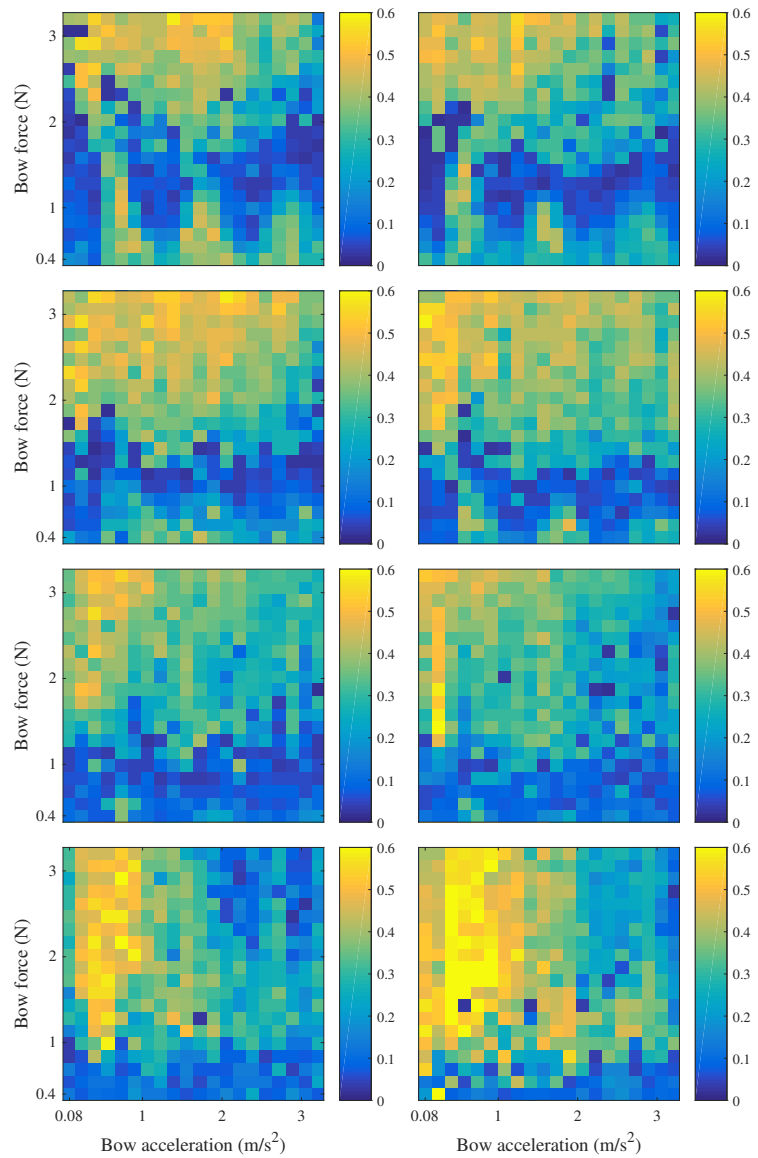

Figure 16: Magnitude of jump in bridge force following the first slip, normalised by the bow force $N$, based on the measurements shown in Fig. 5 and plotted in the Guettler plane for the same 8 cases in the same format as Fig. 15. From top left, $\beta=0.0357,0.0449,0.0566,0.0714,0.0899,0.01133$, $0.1428,0.18$.

such measurements.

A more surprising feature of Fig. 15 is that $\mu_{s}$ depends strongly on the bow's acceleration whereas it is largely unaffected by the bow force: $\mu_{s}$ seems to halve in magnitude from the lowest to the highest values of the acceleration. Thus, although the independence of $\mu_{s}$ from $N$ supports the notion that friction force $f$ is at least roughly proportional to normal force $N$ over most of the studied range, it would appear that additional friction-bearing capability is somehow present at low acceleration, or equivalently at longer sticking time-scales. This observation may be associated with the concept of "junction growth" highlighted in the earlier literature of friction [43, 29]. Once the acceleration is high enough that this enhanced friction has disappeared, however, it can be seen in all cases that the estimated $\mu_{s}$ is lower towards the bottom of each plot, where bow force is low. This may point to a change in the balance between Coulomb's law and Hertzian contact conditions as bow force varies (recall the discussion relating to Fig. 11).

In case there was an influence from the long-term thermal history associated with the sequence of testing, the same measurement was repeated with the force vs acceleration parameter space traversed in the opposite order (results not shown here). A virtually identical result was obtained. This indicates that the results in Fig. 15 are not significantly changed by the order in which the data is gathered. In addition, friction at first slip was similarly measured with a series of constant-velocity bowing gestures instead of constant-acceleration gestures, and a similar dependence of friction on bow velocity was observed. The details of these additional experiments may be found in Galluzzo [32].

Looking at further details in Fig. 15, a relatively weak structure of vertical stripes can be discerned. It appears in similar positions in all cases, more clearly in some cases than others. This feature seems to be related to a pattern evident in the top four cases of Fig. 16, especially at lower bow force. Accelerations associated with unusually high limiting coefficient of friction, in columns 8 and 15 (with values 1.23 and $2.38 \mathrm{~m} / \mathrm{s}^{2}$ ), also seem to produce unusually low values of the initial jump in bridge force.

Figure 16 also shows a strong dependence on bow force: normalised jumps are biggest at high bow force, then in most cases they go through a minimum before recovering somewhat at the lowest forces. The broad conclusion is that the real system shows more complicated behaviour than any of the models: as already explained, friction-curve models always have jumps while the current thermal model never shows them. In the measurements, there are always some regions of the Guettler plane showing significant jumps, and others where jumps are vanishingly small so that the measured bridge force is more reminiscent of the thermal simulations. It is not at present clear what is responsible for this structure, or for the vertical stripes mentioned above, but the data shown here may well provide a sensitive test for any proposed new models.

\subsection{Transients from the Schumacher rig}

Finally, it is illuminating to investigate some results from a different friction experiment. As explained earlier, a rig designed by Schumacher used a rosin-coated glass rod to bow a violin E string [5]. The results from this rig can be used to ex- 
tend what has been learned from the Galluzzo rig, in two different ways. First, the string and "bow" have significantly different properties from those of the Galluzzo rig, allowing an investigation of how well the candidate models reproduce the effects of parametric variation. Second, the Schumacher rig provides information that goes beyond that from the measurements discussed so far: estimates are computed of the friction force and string velocity at the bowed point. These are quantities not readily accessible to direct measurement, and they shed additional light on the physics of friction in a bowed string.

In order to compare results from this rig with simulation, some changes are needed to the models. The properties of the monofilament steel string are significantly different from those of the cello string: the chosen parameter values are listed in Table 1. Those values apply to all simulations to be shown in this section. For the friction modelling, no change is needed to the two friction-curve models since they contain no free parameters. However, as explained in section 2.3, the thermal model requires modification. The evidence of Fig. 9 suggests that any differences in the thickness of the rosin layer can be ignored in the first instance, but Fig. 10 suggests that the different area of contact should be taken into account. The area can be expected to be smaller than for the Galluzzo rig since both rod and string have smaller diameters. However, it will not be as small as might at first be thought on the basis of Hertzian contact of crossed cylinders (see for example Johnson [29]). The string is sufficiently flexible that it will wrap around the rod somewhat, increasing the contact area. A modest reduction has therefore been made in effective contact radius compared to the earlier simulations: all values were listed in Table 2. However, there is no claim that any of these values of contact size are accurately known from direct measurements.

The Schumacher rig does not produce constantacceleration transients with the accuracy of the Galluzzo rig, because of inertia effects of the trolley that carries the rod. However, the actual motion of the trolley can be measured during testing, and that bow-speed profile can be used in the simulation models to give directly comparable predictions. The nominal acceleration is $2 \mathrm{~m} / \mathrm{s}^{2}$, in the middle of the range explored in the earlier Guettler diagrams, and the actual peak acceleration is not very different.

Figures 17 and 18 show results for a single transient, comparing the measurement with the three simulation models. The choice of this particular transient was based on data quality. As has been explained in detail in earlier work [5], the data pro-
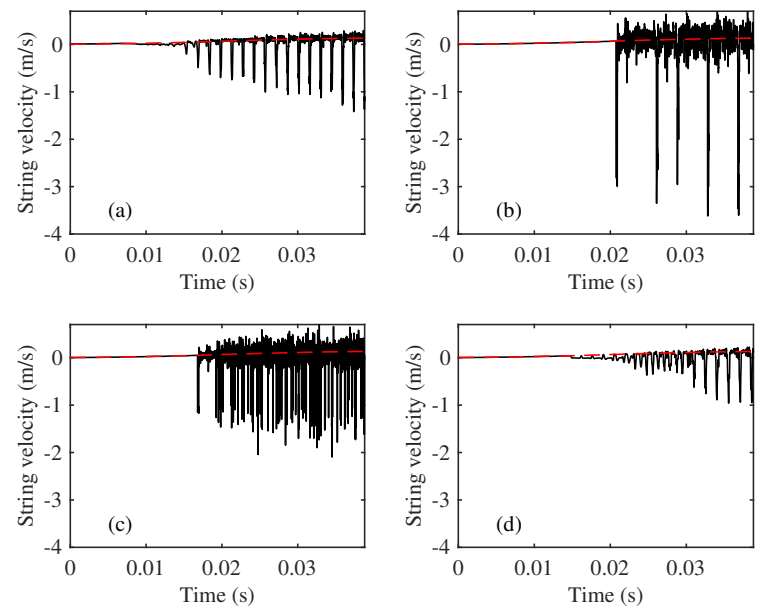

Figure 17: String-centre velocity from a transient: (a) measured by the Schumacher rig; (b) simulated with the classical friction curve model; (c) simulated with the reconstructed friction curve model; (d) simulated with the thermal model. The dashed line shows the velocity of the 'bow'.
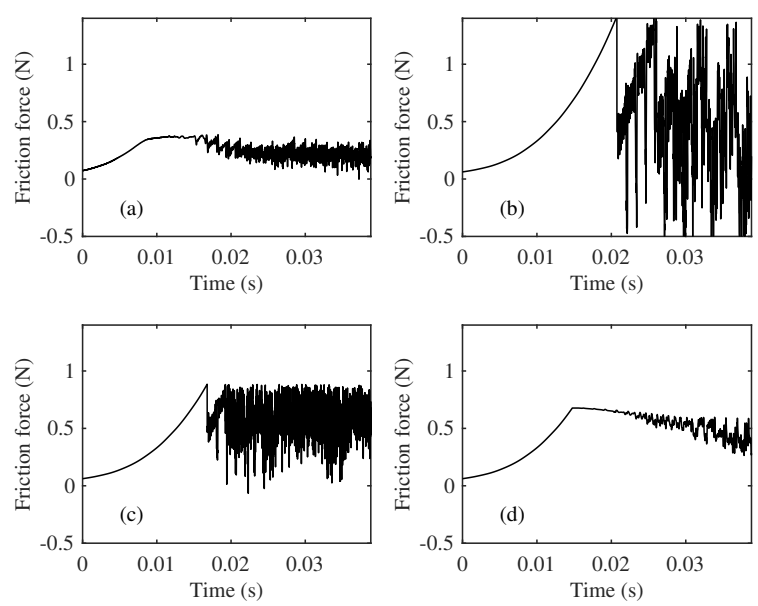

Figure 18: Friction force from the same transients shown in Fig. 17: (a) measured by the Schumacher rig; (b) simulated with the classical friction curve model; (c) simulated with the reconstructed friction curve model; (d) simulated with the thermal model.

cessing used with this rig gives two versions of the reconstructed force and velocity waveforms, and the level of agreement between the two gives a measure of the reliability and accuracy of the measurement and processing. By this measure, the case shown here was one of the best ever produced by the rig. It had a normal force of $1.18 \mathrm{~N}$, and a bowing position with $\beta=0.127$.

The quantity plotted in Fig. 17 is the velocity of the centre of the string at the bowed point: this does not exactly match the trolley velocity during 
sticking because of the effect of torsion, inducing some rolling of the string on the bow. However, the trend tracks the trolley velocity, shown as a dashed line. This particular measured transient shows something close to Guettler's "perfect start" to the note: a single slip per period is a signature of the Helmholtz motion, and that pattern is established more or less from the first slip. None of the simulation models behave so well. Both friction curve models show very irregular and complicated motion within the time range plotted here, bearing no discernible resemblance to the measured waveform. The thermal model is better: it shows Helmholtz motion by the end of the time interval plotted. It is preceded by a Guettler "loose/slipping" transient with a period of double-slipping motion in the early stages, and this transient is sufficiently short (about $15 \mathrm{~ms}$ ) that it would in fact be perceptually acceptable [39].

Figure 18 shows the corresponding waveforms of friction force. For the very early stage of the transient these show a parabolic profile very similar to the bridge force waveforms seen earlier. As already explained, the section of string between bow and bridge behaves quasi-statically before the first slip occurs, and this accounts for the agreement. Later in the evolution of the transient, though, the waveforms of friction force and bridge force are quite different: Fig. 19 shows the bridge force from the same measured transient.

The extreme case of difference between the two waveforms would arise in the idealised situation of steady Helmholtz motion based on a friction-curve law and an ideal string. In that case the friction force would be constant throughout the motion (as first explained by Raman [44]), whereas the bridge force would show the sawtooth waveform familiar from earlier plots. Comparing Figs. 18a and 19 reveals a trace of this behaviour: the friction force shows much more limited variation than the bridge force.

It is clear from Fig. 18 that the classical frictioncurve model (case b) gives a limiting static friction force before the first slip that is far higher than the measurement. It is also a lot higher than for the thermal model (case d) because of the effect of the reduced contact area. Comparing cases a and $\mathrm{d}$, it can be seen that the chosen contact radius has resulted in a maximum force that is broadly comparable with the measured result.

Given the strikingly poor performance of the simulations based on the classical and reconstructed friction curves shown in Figs. 17 and 18, it might be asked whether any friction-curve model can give a satisfactory response for this case. One striking aspect of the force waveforms is that the pre-

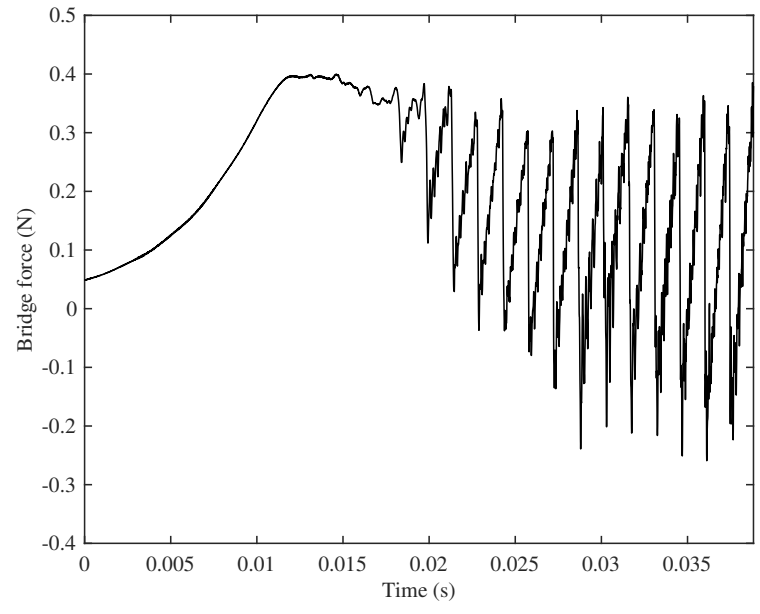

Figure 19: Bridge force from the measured transient shown in Figs. 17(a) and 18(a).

dicted limiting force before the first slip is much bigger than was measured. This is a direct consequence of the assumed coefficients of static friction: see equations $(1,2)$. That suggested a very simple exploratory study: by artificially reducing the normal force in the simulation with the classical friction-curve model, the limiting friction force would be correspondingly reduced. Simulations with a range of forces were tried (not reproduced here), but none of them produced Helmholtz motion within the time-span of these plots. It seems likely that the very low damping of this string model, combined with the inherent "twitchiness" of a friction-curve model, makes for a very "hard-toplay" string. That conclusion follows hints given by the earlier discussion of results from the Galluzzo rig, but the effect is stronger in the present case.

Another aspect of the results from the Schumacher rig has been previously highlighted by Woodhouse et al. [5, 17]. The trajectory can be plotted in the force-velocity plane, and it is usually found to show a hysteresis loop, broadly similar to ones observed in earlier studies of stickslip friction $[45,20]$. The result for this particular bowed transient is shown in Fig. 20, derived from the Helmholtz motion towards the end of the waveforms shown in Figs. 17a and 18a. The patch of "scribble" near a relative sliding velocity of zero corresponds to sticking of the string to the bow: the string centre can still move by rolling on the bow.

Such a hysteresis loop gives direct evidence that no friction-curve model can give a physically correct description. It has already been seen that a hysteresis phenomenon can occur within a frictioncurve model (see Fig. 2), but in that case the individual sampled data points would all lie on the cho- 


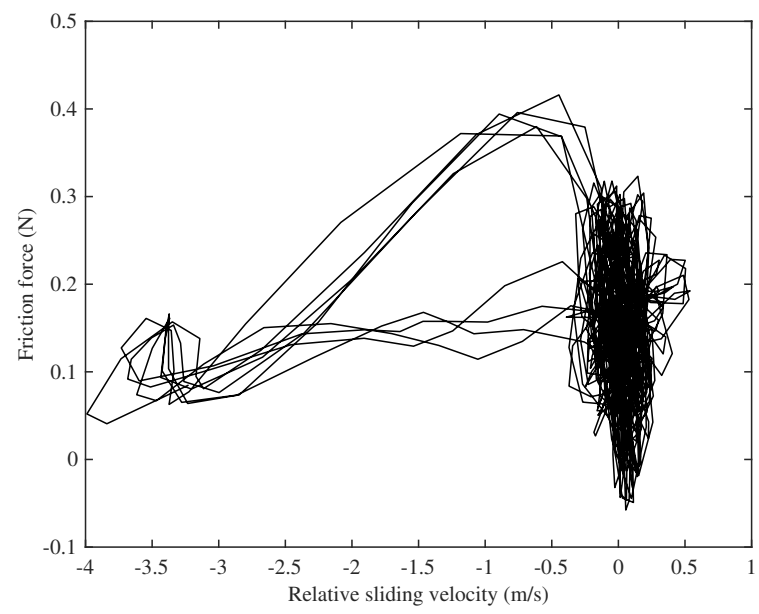

Figure 20: Hysteresis loop of friction force as a function of relative sliding speed between string and bow, from the final stage of the measured bowedstring waveforms shown in Figs. 17a and 18a.

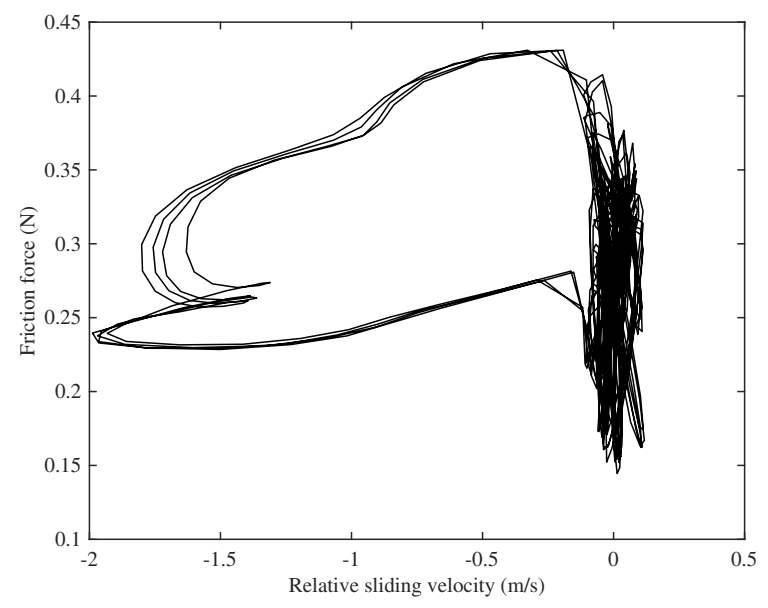

Figure 21: Trajectory of friction force against relative sliding velocity for motion simulated with the thermal model, from the same run as the transients shown in Figs. 17 and 18 but from a later portion where approximately steady Helmholtz motion had been established. It is to be compared with the measurement shown in Fig. 20. the maximum force during a stick-to-slip transition being reached with a significantly non-zero value of relative sliding speed. Before that maximum, there is evidence of creep as the "sticking" portion of the curve bends to the left. This comment may relate to a feature visible in Figs. 18 and 19. This particular transient showed a rounded initial slip with no initial jump, similar to the low-force examples in Fig. 13, suggesting that stick-to-slip transitions were perhaps following the sequence of events described for the thermal model, including some initial creep.

\section{Discussion and conclusions}

In this paper, all available types of experimental data in which a stretched string was bowed by a "rigid" point bow have been examined. A representative selection of the results has been compared systematically with simulations based on the most favoured theoretical models from the existing literature. In the light of this comparison, it is now possible to give an overview of the current state of the art in accurate simulation of bowed-string transients.

There are broadly two types of evidence bearing upon the question of accuracy, which may be termed "physics-based" and "pragmatic". Ideally, one would like a simulation model that was based fully on an understanding of the underlying physics, and supported by direct and independent measurements of the relevant material behaviour and parameter values. The evidence shown here makes it clear that such a model does not yet exist, so it is also of interest to ask the more pragmatic question "can any of the existing models be relied upon to capture at least some aspects of bowed-string behaviour, so as to shed light on issues of interest to a musician?"

A simulation model for a bowed string requires several ingredients. Some of those concern the vibrational behaviour of the strings and the instrument body, and the process of sound radiation by that vibrating body. When this vibration is of sufficiently small amplitude that linear theory can reasonably be applied, a good case can be made that a satisfactory physics-based model is indeed available. Earlier papers $[4,7,8]$ have described a rather complete model, including the various wave-types that can occur in a string, and the interaction between them all and the vibration of the instrument body. The model is complicated, but it is built up from well-studied and uncontroversial ingredients, it can be calibrated by independent measurements, and it has been validated against detailed measure- 
ments of plucked strings.

However, the other main ingredient of a bowedstring model is more problematic: the frictional behaviour at the bow-string contact, mediated by the use of rosin. Stick-slip friction is an inherently nonlinear phenomenon, and it has proved difficult to pin down in a fully satisfactory model; not only for violin rosin, but also in many other areas involving friction-excited vibration, ranging from brake squeal to earthquake dynamics [26]. Many models for friction have been proposed, generally building on physics-based evidence from particular measurements.

In the context of bowed-string dynamics, two main classes of friction model have been discussed. Until relatively recently, all work on the subject assumed some version of the friction-curve model, in which the friction force was assumed to be a function of the instantaneous sliding speed only, with no history dependence. Two representatives of this class of model have been considered here: one based on direct measurement of the friction force from a rosin-coated interface during imposed steady sliding at a range of speeds $[30,20]$, the other inferred from details of the dynamics of actual bowed strings by comparison with theoretical predictions [32].

The third model considered here belongs to a different class, in which history dependence is included by allowing the friction force to depend on one or more internal state variables, each with its own evolution equation. Experimental evidence for the particular case of rosin suggests, very strongly, that a key state variable is the temperature near the contact [20]. This has motivated the development of thermal models of friction, and the leading current contender among these models [18] has been considered here. It is based on the idea that friction force is associated with plastic yield in the rosin layer. The yield stress is allowed to be a function of temperature, chosen by requiring that the model should reproduce the steady-sliding results used in the "classical" friction curve model.

\subsection{Physics-based evidence}

The longest-established physical evidence relating to rosin friction comes from the results of steady-sliding measurements. These underlie both the classical friction-curve model and the thermal model. An appropriate model needs to be consistent with that data, but steady-sliding measurements simply do not provide enough information to be able to design a complete and accurate model. More recently, examples have been published [20, 5] of hysteresis loops in the force-velocity plane such as the one shown earlier (see Fig. 20): such loops definitively show that no friction-curve model can 1415 be physically correct. However, the existence of ${ }_{1416}$ loops does not necessarily mean that the transient ${ }_{1417}$ string motion is sensitively affected by them. It ${ }_{1418}$ should also be noted that the loops do not give ${ }_{1419}$ clear guidance about what alternative model should $\quad{ }_{1420}$ be used: any model involving internal state vari- ${ }_{1421}$ ables and consequent history dependence of friction $\quad{ }_{1422}$ is likely to produce loops in such plots.

Evidence has been shown to indicate a significant $\quad{ }_{1424}$ influence of contact temperature on rosin frictional ${ }_{1425}$ properties [20, 18]: it appears likely that a process 1426 of melt lubrication is involved in stick-slip dynam- ${ }^{1427}$ ics. Additional quantitative evidence can be added ${ }_{1428}$ on this question: Fig. 22 shows an example of a ${ }^{1429}$ standard rheometer measurement (ARES-LC) on a ${ }_{1430}$ bulk sample of violin rosin. This plot shows the ${ }^{1431}$ complex shear modulus as a function of temperature, a representative example of a relevant material property. Because of limitations of the available test methods, rosin could be separately tested in the "solid" state and the "liquid" states, but being a glassy material the transition between the two occurs over a substantial temperature range and it was not possible to test at intermediate temperatures. This explains the gap in the plots, but it is easy to guess more or less how the shear modulus must behave in this gap. Note that the behaviour of the complex modulus follows expectation: predominantly real (i.e. elastic) at lower temperatures, predominantly imaginary (i.e. viscous) at higher temperatures. This plot shows the shear modulus changing by some five orders of magnitude between room temperature and $70^{\circ} \mathrm{C}$. There can be little doubt that this dramatic variation is a key factor in the dynamic frictional behaviour of rosin.

However, it has not yet proved possible to base a successful simulation of bowed strings on a model incorporating this detailed bulk behaviour of rosin. The thermal model used in the studies reported here is more crude, and it is important to acknowledge its assumptions and limitations. The model is not based on independent measurements like those of Fig. 22; instead, it is based on an assumed form of constitutive law employing a single averaged contact temperature, with a temperature-dependent yield stress deduced by fitting to the steady-sliding results. One might guess that the very rapid variation of rosin properties with temperature shown by Fig. 22 will mean that no model based on a single averaged temperature in the contact will in the end be sufficient. There will inevitably be variations of temperature around the contact footprint and through the thickness of the rosin layer, leading to big variations in mechanical properties. For example, it is possible that the balance between "plastic"

6
7
19
1420
22
23




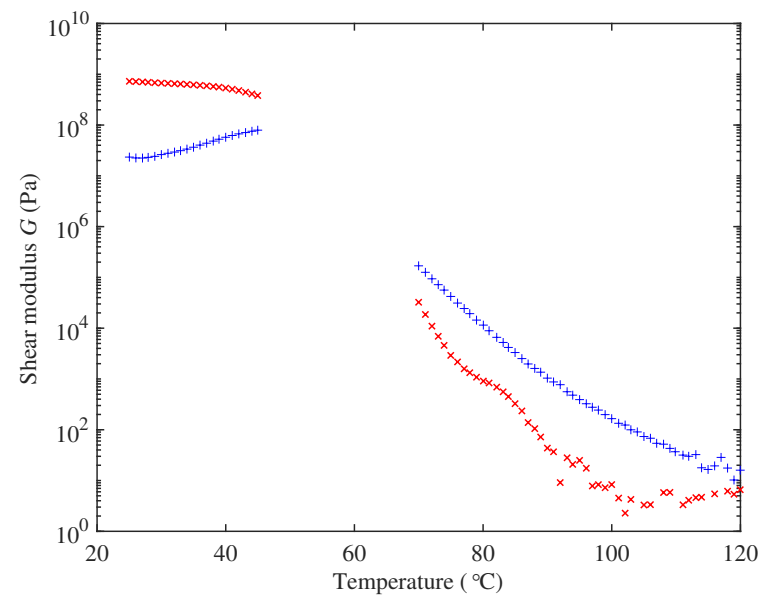

Figure 22: Complex shear modulus $G=G^{\prime}+i G^{\prime \prime}$ of violin rosin, measured as a function of temperature: $\times$ denotes $G^{\prime}$, + denotes $G^{\prime \prime}$. Separate measurements were made for rosin in the solid and liquid states, with a range of intermediate temperatures not accessible to either test. Measurements on the solid sample were at a frequency of $100 \mathrm{rad} / \mathrm{s}$ and a strain of $0.01 \%$, those on the liquid sample at $10 \mathrm{rad} / \mathrm{s}$ and a strain of $1 \%$. and "viscous" behaviour will vary with temperature and hence with position. The detailed processes leading to transitions between sticking and slipping are likely to be sensitive to such variations.

Even the simplified thermal friction model considered here contains parameter values that are not easy to determine with great accuracy: alongside the thermal properties of the substrate materials of string and "bow", the thickness of the rosin layer and the size of the contact footprint are required. These are also needed for the configuration of the steady-sliding measurements, and the values are very likely to have been different in those tests because the contact geometry was different. Finally, in order to probe the Schelleng or Guettler diagrams it is necessary to know how the parameter values vary with normal force. Some evidence has been shown to explore the sensitivity of the predicted string motion to all these factors: see Figs. 9-11.

\subsection{Empirical evidence}

These various uncertainties mean that at present one must fall back on assessing the candidate models based on empirical evidence, since it must be accepted that no current model has a complete and secure basis in physics. There is a long history of assessing bowed-string models in this way, and there are some undoubted success stories. A hun- dred years ago, Raman's original model was already able to give a reasonable match to the wide variety of possible periodic vibration regimes of a bowed string that had been observed [44]. The earliest useful predictions of transient motion came in the 1970s with the development of time-domain simulation methods based on variants of the frictioncurve model [34]. These were able to give accounts of several observed phenomena that were at least qualitatively correct: for example the variation of Helmholtz waveform with bow force, the regime transitions providing the bow-force limits in the Schelleng diagram, the "wolf note", and the fact that a bowed note tends to play flat (i.e. with a longer period) when bow force is increased [34].

The present study has aimed to go further than this, and seek quantitative agreement between experiment and simulation for at least some details of bowed-string transients. Evidence of various kinds has been presented: low-level comparisons of individual transients, and higher-level comparisons of variation within the Schelleng or Guettler diagrams of some computed metrics based on regime identification and transient length. The general impression given by all these comparisons is fairly clear. The classical friction curve model performs consistently worst of the three models tested. The reconstructed friction curve gives a clear improvement in most cases, which is perhaps not too surprising since this model was arrived at by a type of inverse calculation based on measurements of the kind examined here. The thermal model, while clearly disagreeing with measurements in some details, generally comes closest to reality.

\subsection{Consequences for future friction models}

Examining the evidence in more detail gives clues about particular aspects of the existing models that need addressing. For this purpose the high-level information from the Schelleng and Guettler diagrams, although interesting, is often hard to interpret. Details of individual waveforms are more immediately useful. One particular aspect of transient behaviour concerns the behaviour around the moment of first slip. A variety of evidence has been shown here, showing some intriguing details.

First, Fig. 15 showed the maximum value of friction force before first slip, corresponding at least roughly to the concept of coefficient of static friction. All three theoretical models would predict a fixed value for this maximum coefficient of friction, but in fact the experimental results in the Guettler plane showed a very clear trend towards higher force when the timescale of sticking was longer. 
This observation probably links to the concept of "junction growth" highlighted in the earlier literature of friction [43, 29], and also to some of the more recent work on rate-and-state friction models (for example [46]) where a possible physical interpretation of the internal state variable relates to the "age" of a typical asperity contact.

Next, Figs. 13 and 16 reveal some informative details about what happens at the moment of first slip. For all three theoretical models the answer is clear: any friction-curve model predicts jumps determined by the shape of the curve and the magnitude of the normal bow force, while the thermal model does not allow jumps at all so that first slip is always a rather gentle process. The experimental results show behaviour of both kinds, and when viewed in the Guettler plane in Fig. 16 there is significant (and rather unexpected) structure in the jump magnitude. This suggests that a correct model of rosin friction needs to allow something like a brittle-ductile transition, so that under some circumstances an abrupt breakaway can occur, leading to a jump in force, whereas in other circumstances the release is gradual and quite reminiscent of the thermal model predictions. To reproduce the structure revealed in the Guettler plane may pose a stiff challenge for the next generation of friction models. It may be noted that the particular example from the Schumacher rig shown in Fig. 18 showed a transient reminiscent of the thermal model, with a gradual first release. The available data from this rig does not allow a study as comprehensive as Fig. 16, but nevertheless a large number of individual transients have been recorded. When a next-generation friction model is formulated, it may be worth revisiting this data resource for cases to compare.

There is one more noteworthy aspect of behaviour near first slip. All Guettler transients show an initial phase of "sticking", with parabolic growth in friction force. However, when examined in careful detail many of these transients show evidence of some creep before the obvious first slip: the force lags progressively behind the value expected from perfect sticking. This suggests that the rosin shows some viscous-like behaviour during nominal sticking, with details probably dependent on the residual temperature from whatever has happened before the particular transient being examined.

A related effect was seen in the steady Helmholtz motion from the Schumacher rig. The loop plotted in Fig. 20 shows a patch of "scribble" connoting the sticking phase, but when slipping commences the force continues to rise while the curve moves to the left, and the maximum force occurs at a relative slip speed around $0.5 \mathrm{~m} / \mathrm{s}$. Bearing in mind that the thickness of rosin layer in this rig is of the order of microns, the average strain rate through the thickness of rosin implied by this observation is of the order of $10^{5}$ : not at all what one would ordinarily describe as "creep"! This poses a problem of its own for efforts to construct physics-based models: bulk measurements such as those shown in Fig. 22 cannot easily be made at strain rates anywhere near as high as this.

In summary, the evidence suggests that an accurate model for rosin friction needs to be based on temperature, but will be more elaborate than the current model. Ideally, it would be based on a detailed model of physics grounded in independent measurements of rosin properties. It would need to include some allowance for viscous-like behaviour in place of true sticking, and it probably needs to incorporate something like a brittle-ductile transition. One might hope that some of this behaviour will emerge naturally from a model taking account of the detailed variation of temperature and material properties around the contact zone, but to construct and validate such a model could be a difficult undertaking.

It is also possible that some useful improvements could be achieved by a more pragmatic approach, staying closer to the existing model. Perhaps a constitutive model could be formulated such that the single averaged contact temperature currently in use led to at least some of the desired behaviour. As far as it goes, the thermal simulation to calculate this averaged temperature seems well founded in physics and should be reasonably reliable. Such an approach might have advantages for musical synthesis, and if it achieved a good enough match to the detailed measurements it might shed light on some of the questions of playability that originally motivated this study. But there is no doubt that a full physics-based model would be preferable.

\section{Acknowledgements}

The authors thank Bob Schumacher for experimental results and collaborative discussions over many years, Malcolm Mackley and Simon Butler for the measurement shown in Fig. 22, and Clare Gilmour for lending the cello used in the experimental work on the Galluzzo rig. Comments from three anonymous reviewers helped to improve the paper significantly. PMG thanks the Engineering and Physical Sciences Research Council for financial support during this work. HM acknowledges the government of Canada for a Vanier Canada Graduate Scholarship. 


\section{References}

[1] J. Woodhouse, "On the playability of violins. Part II: Minimum bow force and transients," Acustica, vol. 78, no. 3, pp. 137-153, 1993.

[2] R. T. Schumacher and J. Woodhouse, "The transient behaviour of models of bowed-string motion," Chaos: An Interdisciplinary Journal of Nonlinear Science, vol. 5, no. 3, pp. 509523, 1995.

[3] R. T. Schumacher and J. Woodhouse, "Computer modelling of violin playing," Contemporary Physics, vol. 36, no. 2, pp. 79-92, 1995.

[4] J. Woodhouse, "The acoustics of the violin: a review," Reports on Progress in Physics, vol. 77, no. 11, p. 115901, 2014.

[5] J. Woodhouse, R. T. Schumacher, and S. Garoff, "Reconstruction of bowing point friction force in a bowed string," The Journal of the Acoustical Society of America, vol. 108, no. 1, pp. 357-368, 2000.

[6] P. M. Galluzzo and J. Woodhouse, "Highperformance bowing machine tests of bowedstring transients," Acta Acustica united with Acustica, vol. 100, no. 1, pp. 139-153, 2014.

[7] H. Mansour, J. Woodhouse, and G. Scavone, "Enhanced wave-based modelling of musical strings. Part 1: Plucked strings," Acta Acustica united with Acustica, vol. 102, no. 6, pp. 1082-1093, 2016.

[8] H. Mansour, J. Woodhouse, and G. Scavone, "Enhanced wave-based modelling of musical strings. Part 2: Bowed strings," Acta Acustica united with Acustica, vol. 102, no. 6, pp. 10941107, 2016.

[9] H. v. Helmholtz and A. J. Ellis, On the sensations of tone as a physiological basis for the theory of music. New York: Dover Publications, 2d english ed., 1954.

[10] K. Guettler, "Wave analysis of a string bowed to anomalous low frequencies," Journal of the Catgut Acoustical Society, vol. 2, no. 6, pp. 814, 1994.

[11] B. Lawergren, "Harmonics of s motion on bowed strings," The Journal of the Acoustical Society of America, vol. 73, no. 6, pp. 21742179, 1983.

[12] J. C. Schelleng, "The bowed string and the player," The Journal of the Acoustical Society of America, vol. 53, no. 1, pp. 26-41, 1973.
[13] E. Schoonderwaldt, K. Guettler, and 1710 A. Askenfelt, "An empirical investigation ${ }_{1711}$ of bow-force limits in the Schelleng diagram," ${ }_{1712}$ Acta Acustica united with Acustica, vol. 94, ${ }_{1713}$ no. 4, pp. 604-622, 2008.

[14] H. Mansour, J. Woodhouse, and G. Scavone, ${ }_{1715}$ "On minimum bow force for bowed strings," ${ }_{1716}$ Acta Acustica united with Acustica, vol. 103, ${ }_{1717}$ pp. 317-330, 2017.

[15] R. Mores, "Maximum bow force revisited," ${ }_{1719}$ The Journal of the Acoustical Society of Amer- 1720 ica, vol. 140, no. 2, pp. 1162-1171, 2016.

[16] K. Guettler, "On the creation of the Helmholtz 1722 motion in bowed strings," Acta Acustica united ${ }_{1723}$ with Acustica, vol. 88, no. 6, pp. 970-985, 2002. ${ }_{1724}$

[17] R. T. Schumacher, S. Garoff, and J. Wood- 1725 house, "Probing the physics of slip-stick fric- ${ }_{1726}$ tion using a bowed string," The Journal of Ad- ${ }_{1727}$ hesion, vol. 81, no. 1 , pp. 723-750, 2005.

[18] J. Woodhouse, "Bowed string simulation us- 1729 ing a thermal friction model," Acta Acustica ${ }_{1730}$ united with Acustica, vol. 89, no. 2, pp. 355- ${ }_{1731}$ 368, 2003.

[19] J. Woodhouse and A. R. Loach, "Torsional be- ${ }_{1733}$ haviour of cello strings," Acta Acustica united ${ }_{1734}$ with Acustica, vol. 85, no. 5, pp. 734-740, 1999. ${ }_{1735}$

[20] J. H. Smith and J. Woodhouse, "The tribol- 1736 ogy of rosin," Journal of the Mechanics and ${ }_{1737}$ Physics of Solids, vol. 48, no. 8, pp. 1633-1681, ${ }_{1738}$ 2000 .

[21] M. E. McIntyre, R. T. Schumacher, and 1740 J. Woodhouse, "On the oscillations of musi- ${ }_{1741}$ cal instruments," The Journal of the Acousti- ${ }_{1742}$ cal Society of America, vol. 74, no. 5, pp. 1325- ${ }_{1743}$ $1345,1983$.

[22] N. H. Fletcher and T. D. Rossing, The physics 1745 of musical instruments. Springer Verlag, 1998. $\quad{ }_{1746}$

[23] A. Akay, "Acoustics of friction," The Journal 1747 of the Acoustical Society of America, vol. 111, ${ }_{1748}$ no. 4, pp. 1525-1548, 2002.

[24] G. Sheng, Friction-induced Vibrations and 1750 Sound: Principles and Applications. CRC 1751 Press, 2008.

[25] A. H. Benade, Fundamentals of musical acous- 1753 tics. London: Oxford University Press, sec- ${ }_{1754}$ ond ed., 1976.

172

6

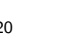

21


[26] J. Woodhouse, T. Putelat, and A. McKay, "Are there reliable constitutive laws for dynamic friction?," Philosophical Transactions of the Royal Society of London. Series A, Mathematical and Physical Sciences, vol. 373, no. 2051, p. 20140401, 2015.

[27] J. O. Smith, Physical audio signal processing: For virtual musical instruments and audio effects. W3K Publishing, 2010.

[28] V. Välimäki, J. Pakarinen, C. Erkut, and M. Karjalainen, "Discrete-time modelling of musical instruments," Reports on Progress in Physics, vol. 69, no. 1, pp. 1-78, 2006.

[29] K. L. Johnson, Contact mechanics. Cambridge, UK: Cambridge University Press, 1985.

[30] H. Lazarus, Die Behandlung der selbsterregten Kippschwingungen der gestrichenen Saite mit Hilfe der endlichen Laplace transformation. Thesis, Technical University of Berlin, 1972.

[31] F. G. Friedlander, "On the oscillations of a bowed string," Mathematical Proceedings of the Cambridge Philosophical Society, vol. 49, no. 3, pp. 516-530, 1953.

[32] P. M. Galluzzo, On the playability of stringed instruments. Thesis, Engineering Department, University of Cambridge, Cambridge, UK, 2003.

[33] M. E. Mclntyre, R. T. Schumacher, and J. Woodhouse, "Aperiodicity in bowed-string motion," Acustica, vol. 49, no. 1, pp. 13-32, 1981.

[34] M. E. McIntyre and J. Woodhouse, "On the fundamentals of bowed string dynamics," Acustica, vol. 43, no. 2, pp. 93-108, 1979.

[35] A. L. Ruina, "Slip instability and state variable friction laws," Journal of Geophysical Research: Solid Earth, vol. 88, no. B12, pp. 10,359-10,370, 1983.

[36] J. R. Rice, N. Lapusta, and K. Ranjith, "Rate and state dependent friction and the stability of sliding between elastically deformable solids," Journal of the Mechanics and Physics of Solids, vol. 49, no. 9, pp. 1865-1898, 2001.

[37] D. C. B. Evans, J. F. Nye, and K. J. Cheeseman, "The kinetic friction of ice," Proceedings of the Royal Society of London. Series A, Mathematical and Physical Sciences, vol. 347, no. 1651, pp. 493-512, 1976.
[38] P. Glendinning, Stability, Instability and 1804 Chaos. Cambridge University Press, $1994 . \quad 1805$

[39] K. Guettler and A. Askenfelt, "Acceptance 1806 limits for the duration of pre-Helmholtz tran- ${ }_{1807}$ sients in bowed string attacks," The Journal ${ }_{1808}$ of the Acoustical Society of America, vol. 101, 1809 no. 5, pp. 2903-2913, 1997.

[40] S. M. Saw, "Influence of finger forces on ${ }_{1811}$ string vibration," thesis, Engineering Depart- ${ }_{1812}$ ment, University of Cambridge, Cambridge, ${ }_{1813}$ UK, 2010.

[41] J. Curtin and T. D. Rossing, "Violin," in The 1815 Science of String Instruments (T. D. Rossing, ${ }_{1816}$ ed.), pp. 209-244, Springer, New York, 2010. ${ }_{1817}$

[42] S. Serafin, The sound of friction: Real-time ${ }_{1818}$ models, playability and musical applications. 1819 Thesis, Music Department, Stanford Univer- ${ }_{1820}$ sity, Stanford, CA, 2004.

[43] D. Tabor, "Junction growth in metallic fric- ${ }_{1822}$ tion: The role of combined stresses and surface ${ }^{1823}$ contamination," Proceedings of the Royal So- ${ }^{1824}$ ciety of London. Series A, Mathematical and ${ }^{1825}$ Physical Sciences, vol. 251, no. 1266, pp. 378- ${ }_{1826}$ 393, 1959.

[44] C. V. Raman, "On the mechanical theory of ${ }_{1828}$ bowed strings and of musical instruments of ${ }^{1829}$ the violin family, with experimental verifica- 1830 tion of results: Part I," Bulletin of the In- ${ }^{1831}$ dian Association for the Cultivation of Sci- ${ }_{1832}$ ence, vol. 15, pp. 1-158, 1918.

[45] P. L. Ko and C. A. Brockley, "The mea- ${ }_{1834}$ surement of friction and friction-induced vi- ${ }_{1835}$ bration," Journal of Lubrication Technology, 1836 vol. 92, no. 4, pp. 543-549, 1970.

[46] T. Baumberger, P. Berthoud, and C. Car- ${ }_{1838}$ oli, "Physical analysis of the state- and rate- 1839 dependent friction law: Dynamic friction," 1840 Physical Review B, vol. 60, no. 6, pp. 3928- ${ }_{1841}$ 3939, 1999.

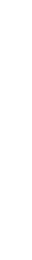

0

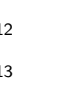

(1)

.

\section{tis}

.

.

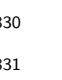

(n)

